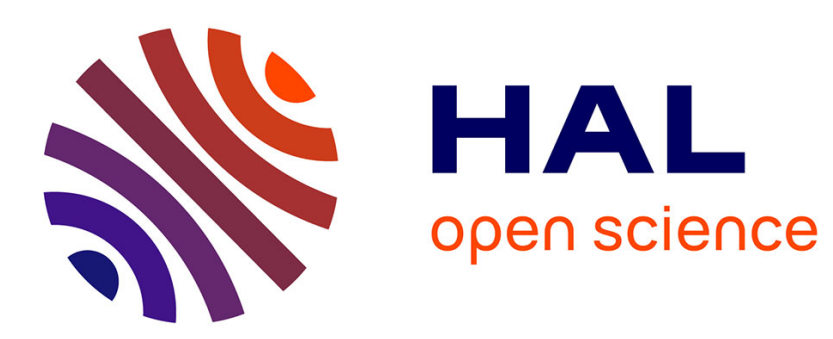

\title{
A numerical framework for modeling flexoelectricity and Maxwell stress in soft dielectrics at finite strains
}

\author{
Julien Yvonnet, Liping Liu
}

\section{To cite this version:}

Julien Yvonnet, Liping Liu. A numerical framework for modeling flexoelectricity and Maxwell stress in soft dielectrics at finite strains. Computer Methods in Applied Mechanics and Engineering, 2017, 313, pp.450 - 482. 10.1016/j.cma.2016.09.007 . hal-01391217

\section{HAL Id: hal-01391217 \\ https://hal.science/hal-01391217}

Submitted on 3 Nov 2016

HAL is a multi-disciplinary open access archive for the deposit and dissemination of scientific research documents, whether they are published or not. The documents may come from teaching and research institutions in France or abroad, or from public or private research centers.
L'archive ouverte pluridisciplinaire HAL, est destinée au dépôt et à la diffusion de documents scientifiques de niveau recherche, publiés ou non, émanant des établissements d'enseignement et de recherche français ou étrangers, des laboratoires publics ou privés. 


\title{
A numerical framework for modeling flexoelectricity and Maxwell stress in soft dielectrics at finite strains
}

\author{
J. Yvonnet ${ }^{1} *$ L.P. Liu ${ }^{2}$ \\ ${ }^{a}{ }^{1}$ Université Paris-Est, Laboratoire Modélisation et Simulation Multi Échelle \\ MSME UMR 8208 CNRS, 5 bd Descartes, F-77454 Marne-la-Vallée, France. \\ b2 Department of Mathematics, Rutgers University, NJ 08854, United States \\ ${ }^{\mathrm{c} 3}$ Department of Mechanical and Aerospace Engineering, Rutgers University, NJ \\ 08854, United States
}

\begin{abstract}
In the present work, a numerical finite element framework is introduced to model and solve the response of nonlinear soft dielectrics, including the effects of Maxwell stress and flexoelectricity at finite strains. Weak forms, finite element discretizations and constistent linearizations, able to handle strain gradient in the context of flexoelectricity are introduced. Numerical algorithms for the treatment of a soft dielectric in a surrounding medium are presented, more specifically to handle the effects of discontinuities of the Maxwell stress at the interfaces. Finally, several benchmarks are proposed to assess the present formulations and numerical schemes, through applications of special cases of interest: induced piezoelectricity in non-piezoelectric materials due to coupling of Maxwell stress and electrets, flexoelectricity, or stretching of electroactive soft dielectrics subjected to an external electric field.
\end{abstract}

Key words: Flexoelectricity, Dielectrics, Finite Elements, Nonlinear dielectrics, Maxwell stress, Finite strains

\section{Introduction}

Soft dielectrics have recently attracted a growing attention due their ability to generate large deformations when they are subjected to an electric voltage.

* Correspondance to J. Yvonnet
Email address: julien.yvonnet@univ-paris-est.fr (J. Yvonnet $\left.{ }^{1}\right)$. 
The induced mechanical deformations caused by the applied electric field can be utilized for sensing and actuation [31,6,43]. As discussed in [42], the electrically induced mechanical deformations are caused by the Maxwell stress. Nonlinear electro-elasticity for soft dielectric elastomers has been discussed in $[12,28]$ and constitutive relation for soft dielectric elastomers have been proposed and discussed by $[41,32,22,36,35]$ for solid and fluid dielectrics. The low dielectric coefficients of soft dielectrics polymers can be increased by addition of reinforcements with high dielectric constants such as ceramic particles or carbon fibres $[13,16,24]$. A review on applications of nonlinear dielectrics to soft actuators, artificial muscles, soft robots and energy harvesting systems can be found e.g. in [17]. In [27], a soft electric generator model of soft dielectric elastomer has been presented.

Moreover, other interesting coupled electromechanical phenomena can occur in soft dielectric and soft biomembranes, like the phenomenon of flexoelectricity. Flexoelectricity describes the coupling between electric polarization and mechanical strain gradient. Even though flexoelectric effects are much larger in ferroelectric materials [9] and complex oxide ceramics [5,25,44,26], the flexoelectricity of several polymers has recently been investigated in [8]. Kogan [20] formulated the first phenomenological theory of flexelectricity and estimated the value range of flexoelectric coefficients. In [10], Deng et al. developed a nonlinear theoretical framework for flexoelectricity in soft materials. In [23], an energy formulation was proposed for continuum electro-elasticity and magneto elasticity. Using the principle of minimum free energy, the EulerLagrange equations of the principle of minimum free energy were derived for a hierarchy of behaviours, including nonlinear dielectric with Maxwell effects and flexoelectricity.

In [33], it was shown how nano composites made of non-piezoelectric components can have an apparent piezoelectric behavior by exploiting the effects of flexoelectricity. In [10] An interesting nonlinear interplay between Maxwell stress and flexoelectricity was described, and the importance of flexoelectricity in soft biological membranes was shown. A prospective on flexoelectricity can be found in [21]. A critical analysis of the current knowledge on the flexoelectricity in common solids can be found in [40].

While many experimental and theoretical studies have been proposed to model soft dielectrics, few numerical works have been proposed so far to model these phenomena in more complex configurations than beams and unidirectional layers. In [2], Aboudi introduced the High-Fidelity Generalized Method of Cells (HFGMC) for prediction of the overall behavior of soft dielectrics composites undergoing large deformations. In [39], an iterative method based on Green's functions was proposed to solve the interior/exterior electrostatic problem for a soft dielectric in a surrounding media. A number of numerical phase-field simulations have been performed to evaluate the effects of flexoelectricity in 
ferroelectrics $[15,38,3,7]$. However, these references resort to finite differences methods with uniform grids, which are limited to very simple geometries and boundary conditions. In [1] Abdollahi et al. introduced a numerical framework based on meshfree method to solve the flexoelectric problem at small strains, where the meshfree approximation was introduced to handle the fourth-order partial differential equations related to strain gradients formulations.

Specific architectures of structures or microstructures could be used to design new soft dielectric systems with higher performances, exploiting the principles described above. Then, numerical methods are required to solve such problems over complex geometries. Motivated by these objectives, we present in this paper a numerical framework for nonlinear dielectrics at finite strain including the coupling due to Maxwell stress and flexoelectricity. The possibility to solve the problem of a soft dielectric in a surrounding media like air is handled by a staggered procedure. Based on the work of [23], the problem of coupled nonlinear flexoelectric problem with Maxwell stress is formulated and discretized by finite elements. The $C^{1}$ required continuity of the displacement field is met by Argyris triangular elements, which allow meshing complex geometries. The consistent linearizations are introduced and the corresponding FEM discretizations are proposed. Finally, benchmarks and applications to induced piezoelectricity in non piezoelectric materials, flexoelectricity and stretching of electroactive polymers are proposed.

The paper is organized as follows. After kinematics and notation preliminaries in section 2, the equations for the nonlinear dielectric problems at finite strains with flexoelectricity are recalled in section 3. Then, the weak forms and consistent linearizations are introduced in sections 4 and 5, respectively. FEM discretizations and a staggered algorithm for dielectrics surrounded by an external media are introduced in sections 6 and 7, respectively. Finally, numerical examples are provided in section 8. Some special cases of interest of this framework, like small strains, Maxwell stress or flexoelectricity effects as separated phenomena are provided in Appendix 11 and their discretization in Appendix 12.

\section{Notations and kinematics preliminary}

Vectors and second order tensors, as well as matrices, are denoted by bold letters A. Third order tensors are denoted by calligraphic uppercase letters $\mathcal{A}$, fourth-order, fifth-order and sixth-order tensors are denoted by double case letters $\mathbb{A}$. Double contraction of indices for second order tensors $\mathbf{A}$ and $\mathbf{B}$ is denoted by $\mathbf{A}: \mathbf{B}=A_{i j} B_{i j}$, dot product for two vectors $\mathbf{a}$ and $\mathbf{b}$ by $\mathbf{a} \cdot \mathbf{b}=a_{i} b_{i}$, and simple contraction of indices for a second order tensor $\mathbf{A}$ and a vector $\mathbf{b}$ is denoted by $(\mathbf{A b})_{i}=A_{i j} b_{j}$. For the purpose of this paper, 
we introduce the triple contraction of indices for two third order tensors $\mathcal{A}$ and $\mathcal{B}$ as: $\mathcal{A} \vdots \mathcal{B}=A_{i j k} B_{i j k}$. The gradient operator is denoted by $\nabla($.$) and$ the divergence operator by $\nabla \cdot($.$) . The third order strain gradient tensor is$ defined by:

$$
(\nabla \boldsymbol{\varepsilon})_{i j k}=\frac{1}{2}\left(\frac{\partial^{2} u_{i}}{\partial x_{j} \partial x_{k}}+\frac{\partial^{2} u_{j}}{\partial x_{i} \partial x_{k}}\right)
$$

For later use, we introduce the following properties. Let a a real-valued vector field and $b$ a scalar field, we have:

$$
\nabla \cdot(\mathbf{a} b)=b \nabla \cdot \mathbf{a}+\mathbf{a} \cdot \nabla b, \quad \frac{\partial}{\partial x_{i}}\left(a_{i} b\right)=\frac{\partial a_{i}}{\partial x_{i}} b+a_{i} \frac{\partial b}{\partial x_{i}} .
$$

For $\mathbf{b}$ a real-valued vector field and $\mathbf{A}$ a second-order tensor field, it can be shown that:

$$
\nabla \cdot(\mathbf{A b})=(\nabla \cdot \mathbf{A}) \cdot \mathbf{b}+\mathbf{A}: \nabla \mathbf{b}
$$

or in indicial notations:

$$
\frac{\partial}{\partial x_{i}}\left(A_{i j} b_{j}\right)=\frac{\partial A_{i j}}{\partial x_{i}} b_{j}+A_{i j} \frac{\partial b_{j}}{\partial x_{i}} .
$$

Let $\mathcal{A}$ a third-order tensor and $\mathbf{B}$ a second-order tensor, then:

$$
\nabla \cdot(\mathcal{A}: \mathbf{B})=(\nabla \cdot \mathcal{A}): \mathbf{B}+\mathcal{A}: \nabla \mathbf{B}
$$

or

$$
\frac{\partial}{\partial x_{k}}\left(A_{i j k} B_{j k}\right)=\frac{\partial A_{i j k}}{\partial x_{k}} B_{j k}+A_{i j k} \frac{\partial B_{j k}}{\partial x_{k}} .
$$

We then introduce the following relations obtained from the divergence theorem:

$$
\begin{aligned}
& \int_{\Omega} \nabla \cdot(\mathbf{a} b) d \Omega=\int_{\partial \Omega} \mathbf{a} \cdot \mathbf{n} b d \Gamma \\
& \int_{\Omega} \nabla \cdot(\mathbf{A b}) d \Omega=\int_{\partial \Omega} \mathbf{n} \cdot \mathbf{A} \mathbf{b} d \Gamma,
\end{aligned}
$$

and

$$
\int_{\Omega} \nabla \cdot(\mathcal{A}: \mathbf{B}) d \Omega=\int_{\partial \Omega} \mathbf{n} \cdot \mathcal{A}: \mathbf{B} d \Gamma .
$$


For later use, we introduce the directional, or Gâteaux derivative of $f(\mathbf{u})$ in the direction of $\mathbf{v}$ defined by:

$$
D_{\mathbf{v}} f(\mathbf{u})=\left[\frac{d}{d \epsilon}\{f(\mathbf{u}+\epsilon \mathbf{v})\}\right]_{\epsilon=0} .
$$

In the context of finite strains analysis, we define $\Omega_{0}$ as the reference configuration and $\mathbf{X}$ and $\mathbf{x}$ material points in the reference and current configurations, respectively. The displacement of a material point is denoted by $\mathbf{u}=\mathbf{x}-\mathbf{X}$.

The deformation gradient tensor is defined by $\mathbf{F}=\nabla_{X} \mathbf{u}+\mathbf{1}$, where $\nabla_{X}($.) denotes gradient with respect to reference configuration, and $\mathbf{C}=\mathbf{F}^{T} \mathbf{F}$ is the right Cauchy-Green strain tensor. The Jacobian is defined as $J=\operatorname{det}(\mathbf{F})$ and $\nabla_{X} \cdot($.$) denotes the divergence operator with respect to reference configura-$ tion. The stain gradient tensor decomposition into dillatation and rotation is denoted by $\mathbf{F}=\mathbf{R} \mathbf{U}, \mathbf{U}=\mathbf{C}^{1 / 2}$.

To study flexoelectricity, we introduce the third-order strain gradient tensor defined by:

$$
\mathcal{G}_{i j k}=\frac{\partial^{2} u_{i}}{\partial X_{j} \partial X_{k}}
$$

Finally, we recall some properties (see e.g. [14]) which will be of interest in the subsequent developments of this paper:

$$
\begin{aligned}
& \frac{\partial J}{\partial \mathbf{F}}=J \mathbf{F}^{-T}, \\
& \left(\frac{\partial \mathbf{F}^{-1}}{\partial \mathbf{F}}\right)_{i j k l}=(\mathbb{F})_{i j k l}=-F_{i k}^{-1} F_{j l}^{-1}, \\
& \left(\frac{\partial \mathbf{F}^{-T}}{\partial \mathbf{F}}\right)_{i j k l}=(\tilde{\mathbb{F}})_{i j k l}=-F_{l i}^{-1} F_{k j}^{-1},
\end{aligned}
$$

and combining (10), (11) and (12),

$$
\begin{aligned}
& \frac{\partial \mathbf{C}^{-1}}{\partial \mathbf{F}}=\mathbb{A} \\
& (\mathbb{A})_{i j k l}=-\left(F_{i k}^{-1} F_{j p}^{-1}+F_{i p}^{-1} F_{j k}^{-1}\right) F_{p l}^{-1}
\end{aligned}
$$

Finally, given $\mathcal{A}$ a general third-order tensor, we have:

$$
\frac{\partial \mathcal{A}_{i j k}}{\partial \mathcal{A}_{l m p}}=\delta_{i l} \delta_{j m} \delta_{k p}
$$




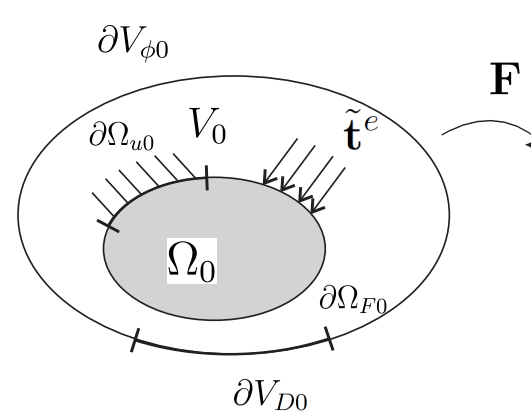

(a)

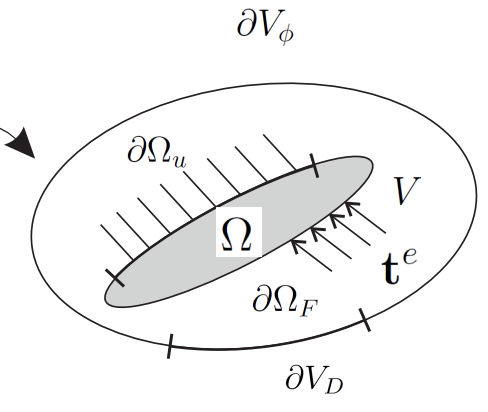

(b)

Figure 1. Solid $\Omega_{0}$ embedded in a surrounding domain $\bar{\Omega}_{0}=V_{0} \backslash \Omega_{0}$ : (a) reference configuration; (b) current configuration.

and for a third-order tensor $\mathcal{A}_{i j k}^{\text {sym }}=\frac{1}{2}\left(\mathcal{A}_{i j k}+\mathcal{A}_{j i k}\right)$ symmetric with respect to indices $i$ and $j$, we have:

$$
\frac{\partial \mathcal{A}_{i j k}^{s y m}}{\partial \mathcal{A}_{l m p}}=\frac{1}{2}\left(\delta_{i l} \delta_{j m}+\delta_{i m} \delta_{j l}\right) \delta_{k p}
$$

\section{Equations for the dielectric problem at finite strains with flexo- electricity}

A domain $V_{0} \in \mathbb{R}^{D}$ is considered in the reference configuration, embedding a solid domain $\Omega_{0}$ and a surrounding media (e.g. air) in a domain $\bar{\Omega}_{0}$ such that $V_{0}=\Omega_{0} \cup \bar{\Omega}_{0}$ as depicted in Fig. 1 . The boundary $\partial \Omega_{0}$ of $\Omega_{0}$ is composed of Dirichlet and Neumann portions, denoted by $\partial \Omega_{0 u}$ and $\partial \Omega_{0 F}$, where displacement and tractions are prescribed respectively, such that $\partial \Omega_{0}=\partial \Omega_{0 u} \cup \partial \Omega_{0 F}$, $\partial \Omega_{0 u} \cap \partial \Omega_{0 F}=\emptyset$. Similarly, the boundary of $V_{0}$ is composed of Dirichlet and Neumann portions, denoted by $\partial V_{0 \phi}$ and $\partial V_{0 D}$, where electric potentials and normal component of electric displacement are prescribed, respectively, such that $\partial V_{0}=\partial V_{0 D} \cup \partial V_{0 \phi}, \partial V_{0 D} \cap \partial V_{0 \phi}=\emptyset$. Counterparts of definitions in the current configuration are defined similarly, omitting the index 0 .

The free energy of the system can be expressed as:

$$
F(\mathbf{u}, \tilde{\mathbf{P}})=U+E^{e l e c}+W^{e x t}
$$

where

$$
U=\int_{\Omega_{0}} \psi d \Omega
$$


$\tilde{\mathbf{P}}$ is the polarization, and $\psi(\mathbf{u}, \tilde{\mathbf{P}})$ is the internal energy given by:

$$
\psi=\psi^{\text {elast }}(\mathbf{U})+\psi^{\text {flexo }}(\mathcal{G}, \tilde{\mathbf{P}})+\psi^{\text {diel }}(\tilde{\mathbf{P}}) .
$$

Moreover, $E^{e l e c}$ is the total electric energy and $W^{\text {mech }}$ is the potential energy of mechanical loadings. The energy $E^{\text {elec }}$ is expressed by:

$$
E^{\text {elec }}=\int_{V_{0}} \frac{\epsilon_{0}}{2} J\left|\mathbf{F}^{-T} \nabla_{X} \phi\right|^{2}-\tilde{\rho}^{e} \phi d \Omega
$$

where $\phi$ is the electric potential, $\epsilon_{0}$ is the vacuum electric permittivity and $\tilde{\rho}^{e}$ is an external charge. Finally, $W^{m e c h}$ is expressed by:

$$
W^{e x t}=-\int_{\partial \Omega_{0 F}} \tilde{\mathbf{t}}^{e} \cdot \mathbf{u} d \Gamma-\int_{\Omega_{0}} \tilde{\mathbf{f}}^{e} \cdot \mathbf{u} d \Omega
$$

where $\tilde{\mathbf{t}}^{e}$ is the applied load and $\tilde{\mathbf{f}}^{e}$ denotes body forces. In (19), $\psi^{\text {elast }}, \psi^{\text {flexo }}$ and $\psi^{\text {diel }}$ are strain energy density functions, whose explicit forms are provided in the sequel. The following relations are introduced:

$$
\begin{aligned}
& \tilde{\mathbf{E}}=-\nabla_{X} \phi, \quad \mathbf{E}=\mathbf{F}^{-T} \tilde{\mathbf{E}}, \\
& \tilde{\mathbf{D}}=J \mathbf{F}^{-1} \mathbf{D}=-\epsilon_{0} J \mathbf{C}^{-1} \nabla_{X} \phi+\mathbf{F}^{-1}\left(\tilde{\mathbf{P}}+\tilde{\mathbf{P}}^{0}\right),
\end{aligned}
$$

where $\tilde{\mathbf{E}}$ the electric current, $\tilde{\mathbf{D}}$ is the electric displacement and $\tilde{\mathbf{P}}^{0}$ is an extrinsic (eigen) polarization. In the following, isotropy is assumed, and then the different strain density functions can be simplified. For example, we choose here for $\Psi^{\text {flexo }}(\mathcal{G})$ associated with the strain gradient (see e.g. [19]):

$$
\psi^{\text {flexo }}(\mathcal{G})=\frac{g}{2} \mathcal{G}_{i k k} \mathcal{G}_{i l l}+f \tilde{P}_{i} \mathcal{G}_{i k k}
$$

where $g$ and $f$ are material constants. The potential $\psi^{\text {diel }}$ is expressed as

$$
\psi^{d i e l}=\frac{1}{2 J}\left(\mathbf{R}^{T} \tilde{\mathbf{P}}\right) \cdot\left(\mathbf{A R}^{T} \tilde{\mathbf{P}}\right)
$$

where $\mathbf{A}$ is the second-order dielectric tensor. We have

$$
\frac{\partial \psi^{\text {diel }}}{\partial \tilde{P}_{m}}=\frac{1}{J} R_{j i} \delta_{j m} A_{i k} R_{j k} \tilde{P}_{j}=\frac{1}{J} R_{m i} A_{i k} R_{j k} \tilde{P}_{j}
$$

or

$$
\frac{\partial \psi^{\text {diel }}}{\partial \tilde{\mathbf{P}}}=\frac{1}{J} \mathbf{R A R}^{T} \tilde{\mathbf{P}}
$$


Using the following property (see [23]):

$$
\frac{\partial \psi}{\partial \tilde{\mathbf{P}}}+\mathbf{F}^{-T} \nabla_{X} \phi=\frac{\partial \psi^{\text {diel }}}{\partial \tilde{\mathbf{P}}}+\frac{\partial \psi^{\text {flexo }}}{\partial \tilde{\mathbf{P}}}+\mathbf{F}^{-T} \nabla_{X} \phi=0,
$$

from (24) and (25) we obtain the following relationship:

$$
\tilde{\mathbf{P}}=-J\left(\mathbf{R A} \mathbf{R}^{T}\right)^{-1}\left[f \mathbf{g}_{X}(\mathbf{u})+\mathbf{F}^{-T} \nabla_{X} \phi\right]
$$

where

$$
\left(g_{X}\right)_{i}(\mathbf{u})=\mathcal{G}_{i k k}=\frac{\partial^{2} u_{i}}{\partial X_{k}^{2}}
$$

For an isotropic dielectric medium, it yields:

$$
\mathbf{A}=\left(\epsilon-\epsilon_{0}\right)^{-1} \mathbf{1}
$$

Then, by the relation $\mathbf{R R}^{T}=\mathbf{1},(29)$ reduces to:

$$
\tilde{\mathbf{P}}=-J\left(\epsilon-\epsilon_{0}\right)\left[f \mathbf{g}_{X}(\mathbf{u})+\mathbf{F}^{-T} \nabla_{X} \phi\right]
$$

and the electric displacement can be expressed by

$$
\tilde{\mathbf{D}}\left(\mathbf{u}, \phi, \tilde{\mathbf{P}}^{0}\right)=-J \epsilon \mathbf{C}^{-1} \nabla_{X} \phi-J f\left(\epsilon-\epsilon_{0}\right) \mathbf{F}^{-1} \mathbf{g}_{X}+\mathbf{F}^{-1} \tilde{\mathbf{P}}_{0}
$$

In this work, we consider a compressible Mooney-Rivlin constitutive model for the dielectric elastomer, defined by the following strain density function:

$$
\psi^{e l a s t}=\frac{\mu}{2}\left[J^{-2 / 3}\left(\lambda_{1}^{2}+\lambda_{2}^{2}+\lambda_{3}^{2}\right)-3\right]+\frac{\kappa}{2}(J-1)^{2},
$$

where $\mu$ and $\kappa$ are the Lamé 's constants such that $\mu=E /(2(1+\nu)), \kappa=$ $E /(3(1-2 \nu))$, with $E$ the Young's mudulus and $\nu$ the Poisson's coefficient, and $\lambda_{\alpha}(\alpha=1,2,3)$ are the principal stretches, i.e. eigenvalues of $\sqrt{\mathbf{C}}$.

The first Piola-Kirchhoff stress tensor $\boldsymbol{\Sigma}=\frac{\partial \psi^{\text {elast }}}{\partial \mathbf{F}}$ is then expressed by

$$
\boldsymbol{\Sigma}=\frac{\mu}{2}\left[-\frac{1}{3} \mathbf{F}^{-T} J^{-2 / 3} \sum_{\alpha} \lambda_{\alpha}^{2}+J^{-2 / 3} \mathbf{F}\right]+\kappa(J-1) J \mathbf{F}^{-T} .
$$

We finally define the third-order tensor $\mathcal{S}$ such that

$$
\mathcal{S}=\frac{\partial \psi^{\text {flexo }}}{\partial \mathcal{G}}
$$


Using the expression of $\psi^{\text {flexo }}$ given in (24) and (15), we obtain:

$$
\mathcal{S}_{i j k}=\left[\frac{g}{2} \mathcal{G}_{i m m}+f \tilde{P}_{i}\right] \delta_{j k}=\left[\frac{g}{2} u_{i, m m}+f \tilde{P}_{i}\right] \delta_{j k}
$$

We define $\tilde{\boldsymbol{\Sigma}}_{M W}$ as the modified Maxwell stress [23], expressed in the solid by:

$$
\tilde{\boldsymbol{\Sigma}}_{M W}=-\frac{\epsilon_{0}}{2} J|\mathbf{E}|^{2} \mathbf{F}^{-T}-\frac{1}{2 J\left(\epsilon-\epsilon_{0}\right)}|\tilde{\mathbf{P}}|^{2} \mathbf{F}^{-T}+\mathbf{E} \otimes \tilde{\mathbf{D}}
$$

In the air, the polarization $\tilde{\mathbf{P}}$ is zero and then the Maxwell stress still exists but reduces to:

$$
\tilde{\Sigma}_{M W}=-\frac{\epsilon_{0}}{2} J|\mathbf{E}|^{2} \mathbf{F}^{-T}+\mathbf{E} \otimes \tilde{\mathbf{D}}
$$

where $\tilde{\mathbf{D}}$ is given by (33).

On the boundary $\partial \Omega_{0}$, we have, as $\boldsymbol{\Sigma}=\mathbf{0}, \mathcal{S}=0$ and $\boldsymbol{\Sigma}_{M W} \neq \mathbf{0}$ in $\bar{\Omega}$ (see [23] for more details):

$$
\boldsymbol{\Sigma} \mathbf{N}-\left(\nabla_{X} \cdot \mathcal{S}\right) \mathbf{N}+\left[\left[\tilde{\boldsymbol{\Sigma}}_{M W}^{\prime}\right]\right] \mathbf{N}-\boldsymbol{\tau}-\tilde{\mathbf{t}}^{e}=0
$$

with

$$
\tau_{p}=\left[\mathcal{S}_{p i j} N_{j}\left(\delta_{i k}-N_{i} N_{k}\right)\right]_{, k}-\left[\mathcal{S}_{p i j} N_{j}-N_{i} N_{k}\right]_{, m} N_{m} N_{k}
$$

By (37) in (41) we see that $\boldsymbol{\tau}=\mathbf{0}$. We summarize the equations of the coupled problem in the following.

The equations of the dielectric problem are given by:

$$
\nabla_{X} \cdot(\tilde{\mathbf{D}})=\tilde{\rho}^{e} \text { in } V_{0}
$$

where $\tilde{\mathbf{D}}$ is given by (33), and, assuming only Dirichlet or Neumann boundary conditions:

$$
\begin{aligned}
& \phi=\phi^{b} \text { on } \partial V_{0 \phi}, \\
& \mathbf{N} \cdot \tilde{\mathbf{D}}=D_{n}^{b} \text { on } \partial V_{D 0} .
\end{aligned}
$$


The equations describing the mechanical problem are given by (see [23]):

$$
\nabla_{X} \cdot(\boldsymbol{\Sigma})-\nabla_{X} \cdot\left(\nabla_{X} \cdot \mathcal{S}\right)+\nabla_{X} \cdot \tilde{\boldsymbol{\Sigma}}_{M W}+\tilde{\mathbf{f}}^{e}=0 \text { in } \Omega_{0},
$$

with boundary conditions

$$
\begin{aligned}
& \boldsymbol{\Sigma} \mathbf{N}-\left(\nabla_{X} \cdot \mathcal{S}\right) \mathbf{N}+\left[\left[\tilde{\boldsymbol{\Sigma}}_{M W}\right]\right] \mathbf{N}-\tilde{\mathbf{t}}^{e}=0 \text { on } \partial \Omega_{F 0}, \\
& \mathbf{u}=\mathbf{u}^{b} \text { on } \partial \Omega_{u 0}, \\
& \mathcal{S} \mathbf{N} \otimes \mathbf{N}=0 \text { on } \partial \Omega_{0} \text { (see [23] for a justification), }
\end{aligned}
$$

where $[[]]=.(.)^{\text {air }}-(.)^{\text {solid }}$. We note that from to the definition (39) and the electro-static equation (42), we also have:

$$
\nabla_{X} \cdot \tilde{\Sigma}_{M W}=0 \text { in } \bar{\Omega}_{0}
$$

and

$$
\nabla_{X} \cdot \tilde{\Sigma}_{M W}=0 \text { in } \Omega_{0}
$$

\section{Weak forms}

To be solved by Finite elements, the above boundary value problem (42)(48) must be recast into weak forms. The relevant details are provided in the following.

\subsection{Dielectric problem}

Let $\phi \in\left\{\phi^{\prime} \mid \phi^{\prime}=\phi^{b}\right.$ on $\left.\partial V_{0 \phi}, \phi^{\prime} \in H^{1}\left(V_{0}\right)\right\}$. Pre-multiplying (42) by a test function $\delta \phi \in\left\{\phi^{\prime} \mid \phi^{\prime}=0\right.$ on $\left.\partial V_{0 \phi}, \phi^{\prime} \in H^{1}\left(V_{0}\right)\right\}$ and integrating over $V_{0}$, we obtain:

$$
\int_{V_{0}} \nabla_{X} \cdot \tilde{\mathbf{D}} \delta \phi d \Omega=\int_{V_{0}} \tilde{\rho}^{e} \delta \phi d \Omega
$$

Using properties (2) and (5) we obtain:

$$
\int_{\partial V_{0}} \tilde{\mathbf{D}} \cdot \mathbf{N} \delta \phi d \Omega-\int_{V_{0}} \tilde{\mathbf{D}} \cdot \nabla_{X}(\delta \phi) d \Omega-\int_{V_{0}} \tilde{\rho}^{e} \delta \phi d \Omega=0 .
$$


Using (44), we obtain, as $\delta \phi=0$ on $\partial V_{0 \phi}$ :

$$
\int_{V_{0}} \tilde{\mathbf{D}} \cdot \nabla_{X}(\delta \phi) d \Omega=\int_{\partial V_{0}} D_{n}^{b} \delta \phi d \Omega+\int_{V_{0}} \tilde{\rho}^{e} \delta \phi d \Omega .
$$

Introducing (33) into the weak form, we obtain:

$$
\begin{aligned}
& \int_{V_{0}} J \epsilon \mathbf{C}^{-1} \nabla_{X} \phi \cdot \nabla_{X}(\delta \phi) d \Omega+\int_{\Omega_{0}} J \mathbf{F}^{-1}\left(\epsilon-\epsilon_{0}\right) f \mathbf{g}_{X} \cdot \nabla_{X}(\delta \phi) d \Omega \\
& =\int_{\Omega_{0}} \mathbf{F}^{-1} \tilde{\mathbf{P}}^{0} \cdot \nabla_{X}(\delta \phi) d \Omega+\int_{V_{0}} \tilde{\rho}^{e} \delta \phi d \Omega+\int_{\partial V_{0 D}} D_{n}^{b} \delta \phi d \Gamma .
\end{aligned}
$$

\subsection{Mechanical problem}

\subsubsection{Dielectric medium without considering a surrounding domain}

In this first case, we consider that boundary conditions i.e. of Dirichlet type (like applied voltage) are directly prescribed over $\partial \Omega_{0}$ and that the surrounding medium can be ignored. In that case, the procedure is the same as in the above, except that the integration is only performed over $\Omega_{0}$. We then have:

$$
\begin{aligned}
& \int_{\Omega_{0}} \nabla_{X} \cdot \boldsymbol{\Sigma} \cdot \delta \mathbf{u} d \Omega-\int_{\Omega_{0}}\left(\nabla_{X} \cdot\left(\nabla_{X} \cdot \mathcal{S}\right)\right) \cdot \delta \mathbf{u} d \Omega \\
& +\int_{\Omega_{0}} \nabla_{X} \cdot \tilde{\boldsymbol{\Sigma}}_{M W} \cdot \delta \mathbf{u} d \Omega+\int_{\Omega_{0}} \tilde{\mathbf{f}}^{e} \cdot \delta \mathbf{u} d \Omega=0 .
\end{aligned}
$$

Using property (3), we obtain:

$$
\begin{aligned}
& \int_{\Omega_{0}} \nabla_{X} \cdot(\boldsymbol{\Sigma} \delta \mathbf{u}) d \Omega-\int_{\Omega_{0}} \mathbf{P}^{\text {elast }}: \delta \mathbf{F} d \Omega \\
& +\int_{\Omega_{0}} \nabla_{X} \cdot\left(\tilde{\boldsymbol{\Sigma}}_{M W} \delta \mathbf{u}\right) d \Omega-\int_{\Omega_{0}} \tilde{\boldsymbol{\Sigma}}_{M W}: \delta \mathbf{F} d \Omega \\
& -\int_{\Omega_{0}} \nabla_{X} \cdot\left(\left(\nabla_{X} \cdot \mathcal{S}\right) \delta \mathbf{u}\right) d \Omega+\int_{\Omega_{0}}\left(\nabla_{X} \cdot \mathcal{S}\right): \delta \mathbf{F} d \Omega+\int_{\Omega_{0}} \tilde{\mathbf{f}}^{e} \cdot \delta \mathbf{u} d \Omega=0 .
\end{aligned}
$$

Using (6):

$$
\begin{aligned}
& \int_{\Omega_{0}} \boldsymbol{\Sigma}: \delta \mathbf{F} d \Omega+\int_{\Omega_{0}} \tilde{\boldsymbol{\Sigma}}_{M W}: \delta \mathbf{F} d \Omega-\int_{\Omega_{0}}\left(\nabla_{X} \cdot \mathcal{S}\right): \delta \mathbf{F} d \Omega \\
& =\int_{\Omega_{0}} \tilde{\mathbf{f}}^{e} \cdot \mathbf{u} d \Omega+\int_{\partial \Omega_{0}}\left[\left[\left[\tilde{\boldsymbol{\Sigma}}_{M W}\right]\right] \mathbf{N}+\mathbf{\Sigma} \mathbf{N}-\left(\nabla_{X} \cdot \mathcal{S}\right) \mathbf{N}\right] \cdot \delta \mathbf{u} d \Omega
\end{aligned}
$$


and then, using (46):

$$
\begin{aligned}
& \int_{\Omega_{0}} \boldsymbol{\Sigma}: \delta \mathbf{F} d \Omega+\int_{\Omega_{0}} \tilde{\boldsymbol{\Sigma}}_{M W}: \delta \mathbf{F} d \Omega-\int_{\Omega_{0}}\left(\nabla_{X} \cdot \mathcal{S}\right): \delta \mathbf{F} d \Omega \\
& =\int_{\Omega_{0}} \tilde{\mathbf{f}}^{e} \cdot \mathbf{u} d \Omega+\int_{\partial \Omega_{0}} \tilde{\mathbf{t}}^{e} \cdot \mathbf{u} d \Omega .
\end{aligned}
$$

Using (4), we have:

$$
\left(\nabla_{X} \cdot \mathcal{S}\right): \delta \mathbf{F}=\nabla_{X} \cdot(\mathcal{S}: \delta \mathbf{F})-\mathcal{S} \vdots \delta \mathcal{G}
$$

with

$$
\delta \mathcal{G}_{i j k}=\left[D_{\delta \mathbf{u}} \mathcal{G}\right]_{i j k}=\frac{\partial^{2} \delta u_{i}}{\partial X_{j} \partial X_{k}} .
$$

Then using (7):

$$
\begin{aligned}
& -\int_{\Omega_{0}}\left(\nabla_{X} \cdot \mathcal{S}\right): \delta \mathbf{F} d \Omega=\int_{\Omega_{0}} \mathcal{S} \vdots \delta \mathcal{G} d \Omega-\int_{\Omega_{0}} \nabla_{X} \cdot(\mathcal{S}: \delta \mathbf{F}) d \Omega . \\
& =\int_{\Omega_{0}} \mathcal{S} \vdots \delta \mathcal{G} d \Omega-\int_{\partial \Omega_{0}} \mathcal{S} \mathbf{N}: \delta \mathbf{F} d \Gamma .
\end{aligned}
$$

By assuming the boundary condition $\mathbf{s}^{\prime}=\mathcal{S} \mathbf{N}=0$ on $\partial \Omega_{0}$, we finally obtain the weak form as:

$$
\int_{\Omega_{0}} \mathbf{P}^{e l a s t}: \delta \mathbf{F}+\tilde{\mathbf{\Sigma}}_{M W}: \delta \mathbf{F}+\mathcal{S} \vdots \delta \mathcal{G} d \Omega=\int_{\Omega_{0}} \tilde{\mathbf{f}}^{e} \cdot \delta \mathbf{u} d \Omega+\int_{\partial \Omega_{0}} \tilde{\mathbf{t}}^{e} \cdot \delta \mathbf{u} d \Gamma \cdot(61)
$$

\subsubsection{Dielectric medium embedded in a surrounding domain}

In this next case, we consider both the solid dielectric and the surrounding domain, as described in section 3. We assume that the surrounding domain (e.g. air) does not have mechanical properties and cannot be polarized, i.e. $\boldsymbol{\Sigma}=0, \tilde{\mathbf{P}}=0, \mathcal{S}=0$, but $\phi \neq 0$ and then $\tilde{\boldsymbol{\Sigma}}_{M W} \neq 0, \forall \mathbf{x} \in \bar{\Omega}_{0}=V_{0} \backslash \Omega_{0}$.

Let $\mathbf{u} \in\left\{\mathbf{v} \mid \mathbf{v}=\mathbf{u}^{b}\right.$ on $\left.\partial \Omega_{0 u}, \mathbf{v} \in H^{1}\left(\Omega_{0}\right)\right\}$. Pre-multiplying (45) by a test function $\delta \mathbf{u} \in\left\{\mathbf{v} \mid \mathbf{v}=0\right.$ on $\left.\partial \Omega_{0 u}, \mathbf{v} \in H^{1}\left(\Omega_{0}\right)\right\}$, and integrating over $V_{0}$ yields:

$$
\int_{\Omega_{0}} \nabla_{X} \cdot \mathbf{\Sigma} \cdot \delta \mathbf{u} d \Omega-\int_{\Omega_{0}}\left(\nabla_{X} \cdot\left(\nabla_{X} \cdot \mathcal{S}\right)\right) \cdot \delta \mathbf{u} d \Omega
$$




$$
+\int_{\Omega_{0}} \nabla_{X} \cdot \tilde{\Sigma}_{M W} \cdot \delta \mathbf{u} d \Omega+\int_{\bar{\Omega}_{0}} \nabla_{X} \cdot \tilde{\Sigma}_{M W} \cdot \delta \mathbf{u} d \Omega+\int_{\Omega_{0}} \tilde{\mathbf{f}}^{e} \cdot \delta \mathbf{u} d \Omega=0
$$

Using property (3), we obtain:

$$
\begin{aligned}
& \int_{\Omega_{0}} \nabla_{X} \cdot(\mathbf{\Sigma} \delta \mathbf{u}) d \Omega-\int_{\Omega_{0}} \boldsymbol{\Sigma}: \delta \mathbf{F} d \Omega \\
& +\int_{\Omega_{0}} \nabla_{X} \cdot\left(\tilde{\boldsymbol{\Sigma}}_{M W} \delta \mathbf{u}\right) d \Omega-\int_{\Omega_{0}} \tilde{\boldsymbol{\Sigma}}_{M W}: \delta \mathbf{F} d \Omega \\
& +\int_{\bar{\Omega}_{0}} \nabla_{X} \cdot\left(\tilde{\boldsymbol{\Sigma}}_{M W} \delta \mathbf{u}\right) d \Omega-\int_{\bar{\Omega}_{0}} \tilde{\boldsymbol{\Sigma}}_{M W}: \delta \mathbf{F} d \Omega \\
& -\int_{\Omega_{0}} \nabla_{X} \cdot\left(\left(\nabla_{X} \cdot \mathcal{S}\right) \delta \mathbf{u}\right) d \Omega+\int_{\Omega_{0}}\left(\nabla_{X} \cdot \mathcal{S}\right): \delta \mathbf{F} d \Omega+\int_{\Omega_{0}} \tilde{\mathbf{f}}^{e} \cdot \delta \mathbf{u} d \Omega=0
\end{aligned}
$$

where $\delta \mathbf{F}=\nabla_{X}(\delta \mathbf{u})$. Using (6), we obtain:

$$
\begin{aligned}
& \int_{\Omega_{0}} \boldsymbol{\Sigma}: \delta \mathbf{F} d \Omega+\int_{\Omega_{0}} \tilde{\boldsymbol{\Sigma}}_{M W}: \delta \mathbf{F} d \Omega+\int_{\bar{\Omega}_{0}} \tilde{\mathbf{\Sigma}}_{M W}: \delta \mathbf{F} d \Omega \\
& -\int_{\Omega_{0}}\left(\nabla_{X} \cdot \mathcal{S}\right): \delta \mathbf{F} d \Omega \\
& =\int_{\Omega_{0}} \tilde{\mathbf{f}}^{e} \cdot \mathbf{u} d \Omega+\int_{\partial \Omega_{0}}\left[\left[\left[\tilde{\boldsymbol{\Sigma}}_{M W}\right]\right] \mathbf{N}+\mathbf{\Sigma} \mathbf{N}-\left(\nabla_{X} \cdot \mathcal{S}\right) \mathbf{N}\right] \cdot \delta \mathbf{u} d \Gamma
\end{aligned}
$$

with $[[]]=.(.)_{\text {solid }}-(.)_{\text {air }}$. Then, using (46), it yields:

$$
\begin{aligned}
& \int_{\Omega_{0}} \boldsymbol{\Sigma}: \delta \mathbf{F} d \Omega+\int_{\Omega_{0}} \tilde{\boldsymbol{\Sigma}}_{M W}: \delta \mathbf{F} d \Omega-\int_{\Omega_{0}}\left(\nabla_{X} \cdot \mathcal{S}\right): \delta \mathbf{F} d \Omega \\
& +\int_{\bar{\Omega}_{0}} \tilde{\boldsymbol{\Sigma}}_{M W}: \delta \mathbf{F} d \Omega=\int_{\Omega_{0}} \tilde{\mathbf{f}}^{e} \cdot \mathbf{u} d \Omega+\int_{\partial \Omega_{0 F}} \tilde{\mathbf{t}}^{e} \cdot \delta \mathbf{u} d \Gamma .
\end{aligned}
$$

Now using (49) and (50) and pre-multiplying by a test function $\delta \mathbf{u} \in\left\{\mathbf{v} \mid \mathbf{v}=0\right.$ on $\left.\partial \Omega_{0 u}, \mathbf{v} \in H^{1}\left(\Omega_{0}\right)\right\}$ and integrating over $V_{0}$ we have:

$$
\int_{\Omega_{0}} \nabla_{X} \cdot \tilde{\Sigma}_{M W} \cdot \delta \mathbf{u} d \Omega+\int_{\bar{\Omega}_{0}} \nabla_{X} \cdot \tilde{\Sigma}_{M W} \cdot \delta \mathbf{u} d \Omega=0 .
$$

Using properties (3), (6) we obtain:

$$
\int_{\Omega_{0}} \tilde{\boldsymbol{\Sigma}}_{M W}: \delta \mathbf{F} d \Omega+\int_{\bar{\Omega}_{0}} \tilde{\boldsymbol{\Sigma}}_{M W}: \delta \mathbf{F} d \Omega=\int_{\partial \Omega_{0}}\left[\left[\tilde{\boldsymbol{\Sigma}}_{M W}\right]\right] \mathbf{N} \cdot \delta \mathbf{u} d \Gamma
$$


Then we obtain from (64):

$$
\begin{aligned}
& \int_{\Omega_{0}}\left(\boldsymbol{\Sigma}-\left(\nabla_{X} \cdot \mathcal{S}\right)\right): \delta \mathbf{F} d \Omega \\
& =\int_{\Omega_{0}} \tilde{\mathbf{f}}^{e} \cdot \delta \mathbf{u} d \Omega+\int_{\partial \Omega_{F}} \tilde{\mathbf{t}}^{e} \cdot \delta \mathbf{u} d \Gamma+\int_{\partial \Omega_{0}}\left[\left[\tilde{\boldsymbol{\Sigma}}_{M W}\right]\right] \mathbf{N} \cdot \delta \mathbf{u} d \Gamma .
\end{aligned}
$$

Using the results of the previous section, we finally obtain the weak form as:

$$
\begin{aligned}
& \int_{\Omega_{0}}(\boldsymbol{\Sigma}: \delta \mathbf{F}+\mathcal{S}: \delta \mathcal{G}) d \Omega \\
& =\int_{\Omega_{0}} \tilde{\mathbf{f}}^{e} \cdot \delta \mathbf{u} d \Omega+\int_{\partial \Omega_{0}} \tilde{\mathbf{t}}^{e} \cdot \delta \mathbf{u} d \Gamma+\int_{\partial \Omega_{0}}\left[\left[\tilde{\boldsymbol{\Sigma}}_{M W}\right]\right] \mathbf{N} \cdot \delta \mathbf{u} d \Gamma
\end{aligned}
$$

Note that in this case, the bulk term $\tilde{\boldsymbol{\Sigma}}_{M W}: \delta \mathbf{F}$ in (66) is converted to external Neumann boundary conditions.

\section{Consistent linearization}

The above problem being highly nonlinear due to both material and geometrical nonlinearities and the presence of the Maxwell stress, we propose a Newton-Raphson procedure to solve it numerically. In that framework, the expression of the different tangent operators related to the above weak forms need to be explicited. In what follows, we provide the different expressions for these operators.

\subsection{Dielectric problem}

By the weak form (54), we set:

$$
\begin{aligned}
& R_{1}(\mathbf{u}, \phi)=\int_{V_{0}} J \epsilon \mathbf{C}^{-1} \nabla_{X} \phi \cdot \nabla_{X}(\delta \phi) d \Omega+\int_{\Omega_{0}} J \mathbf{F}^{-1}\left(\epsilon-\epsilon_{0}\right) f \mathbf{g}_{X} \cdot \nabla_{X}(\delta \phi) d \Omega \\
& -\int_{\Omega_{0}} \mathbf{F}^{-1} \tilde{\mathbf{P}}^{0} \cdot \nabla_{X}(\delta \phi) d \Omega-\int_{V_{0}} \tilde{\rho}^{e} \delta \phi d \Omega-\int_{\partial V_{0 D}} D_{n}^{b} \delta \phi d \Omega .
\end{aligned}
$$

A Taylor expansion of the above residuals gives:

$$
R_{1}\left(\mathbf{u}^{k}+\Delta \mathbf{u}, \phi^{k}+\Delta \phi\right) \simeq
$$




$$
R_{1}\left(\mathbf{u}^{k}, \phi^{k}\right)+D_{\Delta \phi} R_{1}\left(\mathbf{u}^{k}, \phi^{k}\right)+D_{\Delta \mathbf{u}} R_{1}\left(\mathbf{u}^{k}, \phi^{k}\right) .
$$

where we recall that $D_{\mathbf{v}} f(\mathbf{u})$ denotes the directional derivatives defined in (8). The solution for the next increment in an iterative Newton-like procedure consists in solving the linearized problems for $\Delta \phi$ and $\Delta \mathbf{u}$ and to update the field variables for the next iteration through $\phi^{k+1}=\phi^{k}+\Delta \phi, \mathbf{u}^{k+1}=\mathbf{u}^{k}+\Delta \mathbf{u}$. In the following, for the sake of clarity, the superscript $k$ is omitted, then, unless specified, $\mathbf{u} \equiv \mathbf{u}^{k}, \phi \equiv \phi^{k}$.

Results of this linearization procedure are provided below. First, we have trivially:

$$
D_{\Delta \phi} R_{1}(\mathbf{u}, \phi)=\int_{V_{0}} J \epsilon \mathbf{C}^{-1} \nabla_{X}(\Delta \phi) \cdot \nabla_{X}(\delta \phi) d \Omega
$$

Then, let us compute the term $D_{\Delta \mathbf{u}}\left\{J_{\epsilon} \mathbf{C}^{-1} \nabla_{X} \phi \cdot \nabla_{X} \delta \phi\right\}$. We have:

$$
D_{\Delta \mathbf{u}}\left\{J C_{i j}^{-1}\right\}=\frac{\partial J}{\partial F_{k l}} \Delta F_{k l} C_{i j}^{-1}+J \frac{\partial C_{i j}^{-1}}{\partial F_{k l}} \Delta F_{k l}
$$

with

$$
\Delta F_{k l}=\frac{\partial \Delta u_{k}}{\partial X_{l}}
$$

Using (10) and (13) we obtain:

$$
\begin{aligned}
& D_{\Delta \mathbf{u}}\left\{F \in \mathbf{C}^{-1} \nabla_{X} \Delta \phi \cdot \nabla_{X} \delta \phi\right\} \\
& =\nabla_{X i}(\delta \phi) \epsilon J\left\{\left[C_{i j}^{-1} F_{l k}^{-1}+(\mathbb{A})_{i j k l}\right] \nabla \phi_{j}\right\} \Delta F_{k l}
\end{aligned}
$$

which can be re-written as

$$
D_{\Delta \mathbf{u}}\left\{F \epsilon \mathbf{C}^{-1} \nabla_{X} \Delta \phi \cdot \nabla_{X} \delta \phi\right\}=\nabla_{X} \delta \phi \cdot \mathcal{M}_{1}: \Delta \mathbf{F}
$$

with

$$
\left(\mathcal{M}_{1}\right)_{i j k}=\epsilon J\left\{C_{i p}^{-1} F_{j k}^{-1}+(\mathcal{A})_{i p k j}\right\} \nabla \phi_{p}
$$

where we have set $\left(\nabla_{X} \phi\right)_{i} \equiv \nabla_{i} \phi$ and $\left(\nabla_{X} \delta \phi\right)_{i} \equiv \nabla_{i} \delta \phi$ for the sake of clarity. Now let us develop the term:

$$
D_{\Delta \mathbf{u}}\left\{\left(\epsilon-\epsilon_{0}\right) f J \mathbf{F}^{-1} \mathbf{g}_{X} \cdot \nabla_{X} \delta \phi\right\}
$$




$$
=f\left(\epsilon-\epsilon_{0}\right)\left\{\frac{\partial}{\partial F_{k l}}\left\{J F_{i j}^{-1} g_{j} \nabla_{i} \delta \phi\right\} \Delta F_{k l}+\frac{\partial}{\partial \mathcal{G}_{k l m}}\left\{J F_{i j}^{-1} g_{j} \nabla_{i} \delta \phi\right\} \Delta \mathcal{G}_{k l m}\right\}
$$

where we have set $g_{i} \equiv\left(g_{X}\right)_{i}$, with

$$
\mathcal{G}(\Delta \mathbf{u}) \equiv \Delta \mathcal{G}
$$

and

$$
[\Delta \mathcal{G}]_{i j k}=\frac{\partial^{2} \Delta u_{i}}{\partial X_{j} \partial X_{k}}
$$

Using (15), we can show that

$$
\frac{\partial \mathbf{g}_{X}}{\partial \mathcal{G}}=\overline{\mathbb{I}},
$$

with

$$
(\overline{\mathbb{I}})_{i j k l}=\delta_{i j} \delta_{k l} .
$$

The above term can be re-written as

$$
D_{\Delta \mathbf{u}}\left\{\left(\epsilon-\epsilon_{0}\right) f J \mathbf{F}^{-1} \mathbf{g}_{X} \cdot \nabla_{X} \delta \phi\right\}=\nabla_{X} \delta \phi \cdot\left\{\mathcal{M}_{2}: \Delta \mathbf{F}+\mathbb{M}_{3} \vdots \Delta \mathcal{G}\right\}
$$

with

$$
\left(\mathcal{M}_{2}\right)_{i k l}=J f\left(\epsilon-\epsilon_{0}\right)\left\{F_{j i}^{-1} F_{l k}^{-1}+A_{i j k l}\right\} g_{j}
$$

and

$$
\mathbb{M}_{3}=J f\left(\epsilon-\epsilon_{0}\right) \mathbf{F}^{-T} \otimes \mathbf{1} .
$$

In a similar fashion, we obtain

$$
D_{\Delta \mathbf{u}}\left\{\int_{\Omega_{0}} \mathbf{F}^{-1} \tilde{\mathbf{P}}^{0} \cdot \nabla_{X}(\delta \phi) d \Omega\right\}=\int_{\Omega_{0}} \nabla_{X} \delta \phi \cdot \mathcal{M}_{4}: \Delta \mathbf{F} d \Omega
$$

with

$$
\left(\mathcal{M}_{4}\right)_{i k l}=(\mathbb{F})_{i j k l} \tilde{P}_{j}^{0}
$$

We finally obtain the linearized form for the dielectric problem 


$$
\begin{aligned}
& \int_{V_{0}} \nabla_{X} \delta \phi \cdot J \epsilon \mathbf{C}^{-1} \nabla_{X}(\Delta \phi) d \Omega \\
& +\int_{\Omega_{0}} \nabla_{X} \delta \phi \cdot\left(\mathcal{M}_{1}+\mathcal{M}_{2}-\mathcal{M}_{4}\right): \Delta \mathbf{F} d \Omega+\int_{\Omega_{0}} \nabla_{X} \delta \phi \cdot \mathbb{M}_{3} \vdots \Delta \mathcal{G} d \Omega \\
& =-R_{1}\left(\mathbf{u}^{k}, \phi^{k}\right) .
\end{aligned}
$$

\subsection{Mechanical problem}

\subsubsection{Dielectric medium without considering a surrounding domain}

In this section, we first consider a the case where Dirichlet boundary conditions are prescribed on the boundary $\partial \Omega_{0}$ (e.g. direct applied voltage) and where the surrounding domain is not modeled. In that case, we set:

$$
\begin{aligned}
& R_{2}(\mathbf{u}, \phi)=\int_{\Omega_{0}} \boldsymbol{\Sigma}: \delta \mathbf{F}+\tilde{\boldsymbol{\Sigma}}_{M W}: \delta \mathbf{F}+\mathcal{S}: \delta \mathcal{G} d \Omega \\
& -\int_{\Omega_{0}} \tilde{\mathbf{f}}^{e} \cdot \delta \mathbf{u} d \Omega-\int_{\partial \Omega_{0}} \tilde{\mathbf{t}}^{e} \cdot \delta \mathbf{u} d \Gamma .
\end{aligned}
$$

Similarly, the nonlinear problem (66) has to be linearized:

$$
\begin{aligned}
& R_{2}\left(\mathbf{u}^{k}+\Delta \mathbf{u}, \phi^{k}+\Delta \phi\right) \simeq \\
& R_{2}\left(\mathbf{u}^{k}, \phi^{k}\right)+D_{\Delta \mathbf{u}} R_{2}\left(\mathbf{u}^{k}, \phi^{k}\right)+D_{\Delta \phi} R_{2}\left(\mathbf{u}^{k}, \phi^{k}\right) .
\end{aligned}
$$

We can first express:

$$
D_{\Delta \phi} R_{2}=\int_{\Omega_{0}} \delta \mathbf{F}: \frac{\partial \tilde{\boldsymbol{\Sigma}}_{M W}}{\partial \nabla_{X} \phi} \nabla_{X}(\Delta \phi)+\delta \mathcal{G} \vdots \frac{\partial \mathcal{S}}{\partial \nabla_{X} \phi} \nabla_{X}(\Delta \phi) d \Omega
$$

From (37) and (32) we obtain:

$$
\frac{\partial \mathcal{S}_{i j k}}{\partial \nabla \phi_{l}}=-J f\left(\epsilon-\epsilon_{0}\right) F_{l i}^{-1} \delta_{j k}
$$

Now let us compute the second term in (87), using (38), we obtain after some calculations:

$$
\frac{\partial}{\partial \nabla \phi_{k}}\left\{\frac{-\epsilon_{0}}{2} J|\mathbf{E}|^{2} F_{j i}^{-1}\right\}=\left(\mathcal{Q}_{2}^{A}\right)_{i j k}=\epsilon J F_{j i}^{-1} F_{k m}^{-1} E_{m}
$$


Next, we obtain:

$$
\frac{\partial}{\partial \nabla \phi_{k}}\left\{-\frac{\tilde{P}_{m} \tilde{P}_{m}}{2 J\left(\epsilon-\epsilon_{0}\right)} F_{j i}^{-1}\right\}=-\frac{1}{J\left(\epsilon-\epsilon_{0}\right)} \frac{\partial \tilde{P}_{m}}{\partial \nabla \phi_{k}} \tilde{P}_{m} F_{j i}^{-1} .
$$

From (32) we obtain:

$$
\frac{\partial \tilde{P}_{i}}{\partial \nabla \phi_{k}}=-J\left(\epsilon-\epsilon_{0}\right) F_{k i}^{-1}
$$

Then

$$
\frac{\partial}{\partial \nabla \phi_{k}}\left\{-\frac{\tilde{P}_{m} \tilde{P}_{m}}{2 J\left(\epsilon-\epsilon_{0}\right)} F_{j i}^{-1}\right\}=\left(\mathcal{Q}_{2}^{B}\right)_{i j k}=F_{j i}^{-1} F_{k m}^{-1} \tilde{P}_{m} .
$$

Furthermore,

$$
\frac{\partial}{\partial \nabla \phi_{k}}\left\{E_{i} \tilde{D}_{j}\right\}=\frac{\partial E_{i}}{\partial \nabla \phi_{k}} \tilde{D}_{j}+E_{i} \frac{\partial \tilde{D}_{j}}{\partial \nabla \phi_{k}}
$$

From (33) we have:

$$
\frac{\partial \tilde{D}_{i}}{\partial \nabla \phi_{k}}=-\epsilon J C_{i k}^{-1}
$$

Then we obtain

$$
\frac{\partial}{\partial \nabla \phi_{k}}\left\{E_{i} \tilde{D}_{j}\right\}=\left(\mathcal{Q}_{2}^{C}\right)_{i j k}=-F_{k i}^{-1} \tilde{D}_{j}-\epsilon J E_{i} C_{j k}^{-1} .
$$

Finally

$$
\frac{\partial \tilde{\Sigma}_{M W}}{\partial \nabla_{X} \phi}=\mathcal{Q}_{2}
$$

with $\mathcal{Q}_{2}=\mathcal{Q}_{2}^{A}+\mathcal{Q}_{2}^{B}+\mathcal{Q}_{2}^{C}$. Now let us perform the linearization of $R_{2}$ with respect to $\Delta \mathbf{u}$. First,

$$
D_{\Delta \mathbf{u}}\{\boldsymbol{\Sigma}: \delta \mathbf{F}\}=\delta \mathbf{F}: \mathbb{C}^{e l}: \Delta \mathbf{F}
$$

with

$$
\mathbb{C}^{e l}=\frac{\partial \mathbf{\Sigma}}{\partial \mathbf{F}} .
$$

In addition, we have:

$$
D_{\Delta \mathbf{u}}(\mathcal{S} \vdots \mathcal{G}(\delta \mathbf{u}))=\mathcal{G}(\delta \mathbf{u}) \vdots \frac{\partial \mathcal{S}}{\partial \mathcal{G}} \vdots \Delta \mathcal{G}
$$


where

$$
\Delta \mathcal{G}_{i j k}=\frac{\partial \Delta u_{i}}{\partial x_{j} \partial x_{k}}
$$

and

$$
\left(\frac{\partial \mathcal{S}}{\partial \mathcal{G}}\right)_{i j k l m p}=(\mathbb{T})_{i j k l m p}=g \delta_{i l} \delta_{j k} \delta_{m p}
$$

Now let us express

$$
\begin{aligned}
& \frac{\partial \tilde{\boldsymbol{\Sigma}}_{M W}}{\partial \mathbf{F}}=\frac{\partial}{\partial \mathbf{F}}\left\{-\frac{\epsilon_{0}}{2} J|\mathbf{E}|^{2} \mathbf{F}^{-T}\right\}+\frac{\partial}{\partial \mathbf{F}}\left\{\frac{-|\tilde{\mathbf{P}}|^{2} \mathbf{F}^{-T}}{2 J\left(\epsilon-\epsilon_{0}\right)}\right\} \\
& +\frac{\partial}{\partial \mathbf{F}}\{\mathbf{E} \otimes \tilde{\mathbf{D}}\}=\mathbb{Q}_{3}^{A}+\mathbb{Q}_{3}^{B}+\mathbb{Q}_{3}^{C} .
\end{aligned}
$$

Using

$$
\frac{\partial E_{i}}{\partial F_{k l}}=-(\tilde{\mathbb{F}})_{i j k l} \nabla \phi_{j}
$$

and

$$
\frac{\partial \tilde{P}_{i}}{\partial F_{k l}}=-\left(\epsilon-\epsilon_{0}\right) J\left[F_{j i}^{-1} F_{l k}^{-1}+(\tilde{\mathbb{F}})_{i j k l}\right] \nabla \phi_{j}
$$

we obtain, after tedious calculations:

$$
\left(\mathbb{Q}_{3}^{A}\right)_{i j k l}=-\frac{\epsilon J}{2}\left\{F_{j i}^{-1} F_{l k}^{-1} E_{m}^{2}-2(\tilde{\mathbb{F}})_{m p k l} \nabla \phi_{p} E_{m} F_{j i}^{-1}+E_{m}^{2}(\tilde{\mathbb{F}})_{i j k l}\right\}
$$

and

$$
\begin{aligned}
& \left(\mathbb{Q}_{3}^{B}\right)_{i j k l}=\frac{1}{2 J\left(\epsilon-\epsilon_{0}\right)}\left[F_{j i}^{-1} F_{l k}^{-1} \tilde{P}_{m}^{2}\right. \\
& \left.-2\left(\epsilon-\epsilon_{0}\right) J\left(F_{p m}^{-1} F_{l k}^{-1}+(\tilde{\mathbb{F}})_{m p k l}\right) \nabla \phi_{p} \tilde{P}_{m} F_{j i}^{-1}+\tilde{P}_{m}^{2}(\tilde{\mathbb{F}})_{i j k l}\right] .
\end{aligned}
$$

Furthermore,

$$
\frac{\partial \tilde{D}_{i}}{\partial F_{k l}}=-\epsilon_{0}\left\{\frac{\partial J}{\partial F_{k l}} C_{i j}^{-1} \nabla \phi_{j}+J A_{i j k l} \nabla \phi_{j}\right\}+\frac{\partial F_{i j}^{-1}}{\partial F_{k l}} \tilde{P}_{j}+F_{i j}^{-1} \frac{\partial \tilde{P}_{j}}{\partial F_{k l}} .
$$


Then

$$
\frac{\partial}{\partial F_{k l}}\left(E_{i} \tilde{D}_{j}\right)=\left(\mathbb{Q}_{3}^{C}\right)_{i j k l}=-(\tilde{\mathbb{F}})_{i p k l} \nabla \phi_{p} \tilde{D}_{j}+E_{i} \frac{\partial \tilde{D}_{j}}{\partial F_{k l}}
$$

with

$$
\frac{\partial \tilde{D}_{i}}{\partial F_{k l}}=\epsilon_{0} J\left(C_{i j}^{-1} F_{l k}^{-1}+(\mathbb{A})_{i j k l}\right) \nabla \phi_{j}+(\tilde{\mathbb{F}})_{i j k l}\left(\tilde{P}_{j}+\tilde{P}_{0 j}\right)+F_{i j}^{-1} \frac{\partial \tilde{P}_{j}}{\partial F_{k l}} .
$$

Finally, we set

$$
\frac{\tilde{\boldsymbol{\Sigma}}_{M W}}{\partial \mathcal{G}}=\mathbb{R}^{A}+\mathbb{R}^{B}
$$

with

$$
\mathbb{R}^{A}=f \mathbf{F}^{-T} \otimes \mathbf{1} \otimes \tilde{\mathbf{P}}
$$

and

$$
\mathbb{R}^{B}=-J f\left(\epsilon-\epsilon_{0}\right) \mathbf{E} \otimes \mathbf{F}^{-T} \otimes \mathbf{1} .
$$

Finally, the linearized form associated with the mechanical problem is given by:

$$
\begin{aligned}
& \int_{\Omega_{0}} \mathcal{G}(\delta \mathbf{u}) \vdots \mathbb{Q}_{1} \cdot \nabla_{X}(\Delta \phi) d \Omega+\int_{\Omega_{0}} \delta \mathbf{F}: \mathcal{Q}_{2} \cdot \nabla_{X}(\Delta \phi) d \Omega \\
& +\int_{\Omega_{0}} \delta \mathbf{F}: \mathbb{C}^{e l}: \Delta \mathbf{F} d \Omega+\int_{\Omega_{0}} \mathcal{G}(\delta \mathbf{u}) \vdots \mathbb{T} \vdots \Delta \mathcal{G} d \Omega \\
& +\int_{\Omega_{0}} \delta \mathbf{F}:\left(\mathbb{Q}_{3}: \Delta \mathbf{F}+\mathbb{R} \vdots \Delta \mathcal{G}\right) d \Omega=-R_{2}\left(\mathbf{u}^{k}, \phi^{k}\right)
\end{aligned}
$$

with $\mathbb{R}^{(5)}=\mathbb{R}^{A}+\mathbb{R}^{B}$ and $\mathbb{Q}_{3}=\mathbb{Q}_{3}^{A}+\mathbb{Q}_{3}^{B}+\mathbb{Q}_{3}^{C}$.

\subsection{Dielectric embedded in a surrounding media}

If a surrounding media is taken into account, we define the new residual:

$$
\begin{aligned}
& \hat{R}_{2}\left(\mathbf{u}_{n+1}^{k}, \mathbf{u}_{n}, \phi_{n+1}^{k}\right)=\int_{\Omega_{0}} \boldsymbol{\Sigma}\left(\mathbf{u}_{n+1}^{k}, \phi_{n+1}^{k}\right): \delta \mathbf{F}+\mathcal{S}\left(\mathbf{u}_{n+1}^{k}, \phi_{n+1}^{k}\right) \vdots \delta \mathcal{G} d \Omega \\
& \int_{\Omega_{0}} \tilde{\mathbf{f}}^{e} \cdot \delta \mathbf{u} d \Omega-\int_{\partial \Omega_{0}} \tilde{\mathbf{t}}^{e} \cdot \delta \mathbf{u} d \Gamma-\int_{\partial \Omega_{0}}\left[\left[\tilde{\boldsymbol{\Sigma}}_{M W}\left(\mathbf{u}_{n}\right)\right]\right] \mathbf{N} \cdot \delta \mathbf{d} \Gamma
\end{aligned}
$$


The purpose of the above special form is to decouple the application of the discontinous Maxwell term $\left[\left[\tilde{\boldsymbol{\Sigma}}_{M W}\left(\mathbf{u}_{n}\right)\right]\right] \mathbf{N}$ by prescribing it as forces assumed known from the previous evolution increment $n$ on $\partial \Omega_{0}$ at evolution increment $n+1$. The staggered scheme is described in section 7 . In that case, the new linearized problem to be solved is defined as:

$$
\begin{aligned}
& \int_{\Omega_{0}} \mathcal{G}(\delta \mathbf{u}) \vdots \mathbb{Q}_{1}\left(\mathbf{u}_{n+1}^{k}, \phi_{n+1}^{k}\right) \cdot \nabla_{X}(\Delta \phi) d \Omega \\
& \int_{\Omega_{0}} \delta \mathbf{F}: \mathbb{C}^{e l}\left(\mathbf{u}_{n+1}^{k}, \phi_{n+1}^{k}\right): \Delta \mathbf{F} d \Omega+\int_{\Omega_{0}} \mathcal{G}(\delta \mathbf{u}) \vdots \mathbb{T}: \Delta \mathcal{G} d \Omega \\
& =-\hat{R}_{2}\left(\mathbf{u}_{n+1}^{k}, \mathbf{u}_{n}, \phi_{n+1}^{k}\right) .
\end{aligned}
$$

\section{Discretization}

In the following, we present the developments associated with the Finite Element discretization of the above problem. For the sake of simplicity, we restrict the developments to $2 \mathrm{D}$, even though extension to $3 \mathrm{D}$ is straightforward. A mesh of elements is constructed over the domain $V_{0}$, containing $N_{e}$ elements.

We first consider the fully-nonlinear problem with all couplings. The case of small small strains is treated in Appendix 12. The electric potential, test function and potential increment are approximated by:

$$
\phi=\mathbf{N}_{\phi} \phi^{e}, \quad \delta \phi=\mathbf{N}_{\phi} \delta \phi^{e}, \quad \Delta \phi=\mathbf{N}_{\phi} \Delta \phi^{e},
$$

with

$$
\mathbf{N}_{\phi}=\left[N_{1}(\mathbf{x}) ; N_{2}(\mathbf{x}) ; \ldots ; N_{n}(\mathbf{x})\right]
$$

where $N_{I}(\mathbf{x})$ is the shape function of node $I$ in one element, with $n$ the number nodes in one element $e$ and $I=1,2, \ldots, n$ denotes the index of the node. Above, $\phi^{e}$ and $\Delta \phi^{e}$ are column vectors of size $n$. The gradient of the electric potential increment and of the related test functions are expressed by:

$$
\nabla_{X}(\Delta \phi)=\mathbf{B}_{\phi} \Delta \phi^{e}, \quad \nabla_{X}(\delta \phi)=\mathbf{B}_{\phi} \delta \phi^{e}
$$

where the matrix $\mathbf{B}_{\phi}$ is expressed by:

$$
\mathbf{B}_{\phi}=\left[\mathbf{D}_{\phi}^{(1)} ; \mathbf{D}_{\phi}^{(2)} ; \ldots ; \mathbf{D}_{\phi}^{(n)}\right]
$$


and

$$
\mathbf{D}_{\phi}^{(I)}=\left[\begin{array}{c}
\frac{\partial N_{I}(\mathbf{x})}{\partial x_{1}} \\
\frac{\partial N_{I}(\mathbf{x})}{\partial x_{2}}
\end{array}\right]
$$

Next, we introduce the related displacement discretization through:

$$
\mathbf{u}=\mathbf{N}_{u} \mathbf{u}^{e}, \quad \delta \mathbf{u}=\mathbf{N}_{u} \delta \mathbf{u}^{e}, \quad \Delta \mathbf{u}=\mathbf{N}_{u} \Delta \mathbf{u}^{e}
$$

with

$$
\mathbf{N}_{u}=\left[\mathbf{N}_{u}^{(1)} ; \mathbf{N}_{u}^{(2)} ; \ldots ; \mathbf{N}_{u}^{(n)}\right], \quad \mathbf{N}_{u}^{(I)}=\left[\begin{array}{ll}
N_{I}(\mathbf{X}) & 0 \\
0 & N_{I}(\mathbf{X})
\end{array}\right]
$$

Then, we define the vector $[\Delta \mathbf{F}]$ associated with the tensor $\Delta \mathbf{F}$ :

$$
[\Delta \mathbf{F}]=\left[\begin{array}{c}
\frac{\partial \Delta u_{1}}{\partial X_{1}} \\
\frac{\partial \Delta u_{1}}{\partial X_{2}} \\
\frac{\partial \Delta u_{2}}{\partial X_{1}} \\
\frac{\partial \Delta u_{2}}{\partial X_{2}}
\end{array}\right]
$$

which can be related to nodal displacement increments $\Delta \mathbf{u}^{e}$ and test functions in the element $e$ through:

$$
[\Delta \mathbf{F}]=\mathbf{B}_{u} \Delta \mathbf{u}^{e}, \quad[\delta \mathbf{F}]=\mathbf{B}_{u} \delta \mathbf{u}^{e},
$$

where

$$
\mathbf{B}_{u}=\left[\mathbf{D}_{u}^{(1)} ; \mathbf{D}_{u}^{(2)} ; \ldots ; \mathbf{D}_{u}^{(n)}\right]
$$

with

$$
\mathbf{D}_{u}^{(I)}=\left[\begin{array}{ll}
\frac{\partial N_{I}(\mathbf{X})}{\partial X_{1}} & 0 \\
\frac{\partial N_{I}(\mathbf{X})}{\partial X_{2}} & 0 \\
0 & \frac{\partial N_{I}(\mathbf{x})}{\partial X_{1}} \\
0 & \frac{\partial N_{I}(\mathbf{x})}{\partial X_{2}}
\end{array}\right]
$$

Now we introduce the vector associated with the $2 \mathrm{D}$ nonsymmetric compo- 
nents of $\mathcal{G}$ :

$$
[\mathcal{G}(\Delta \mathbf{u})]=\left[\begin{array}{c}
G_{111}(\Delta \mathbf{u}) \\
G_{112}(\Delta \mathbf{u}) \\
G_{211}(\Delta \mathbf{u}) \\
G_{122}(\Delta \mathbf{u}) \\
G_{212}(\Delta \mathbf{u}) \\
G_{222}(\Delta \mathbf{u})
\end{array}\right]=\left[\begin{array}{c}
\frac{\partial^{2} \Delta u_{1}}{\partial X_{1}^{2}} \\
\frac{\partial^{2} \Delta u_{1}}{\partial X_{1} \partial X_{2}} \\
\frac{\partial^{2} \Delta u_{2}}{\partial X_{1}^{2}} \\
\frac{\partial^{2} \Delta u_{1}}{\partial X_{1}^{2}} \\
\frac{\partial^{2} \Delta u_{2}}{\partial X_{1} \partial X_{2}} \\
\frac{\partial^{2} \Delta u_{2}}{\partial X_{2}^{2}}
\end{array}\right] .
$$

Then the associated vectors $[\Delta \mathcal{G}]$ and $[\delta \mathcal{G}]$ are related to displacement increments and test functions through:

$$
[\Delta \mathcal{G}(\mathbf{u})]=\hat{\mathbf{B}}_{u} \Delta \mathbf{u}^{e}, \quad[\delta \mathcal{G}(\mathbf{u})]=\hat{\mathbf{B}}_{u} \delta \mathbf{u}^{e}
$$

where

$$
\hat{\mathbf{B}}_{u}=\left[\hat{\mathbf{D}}_{u}^{(1)} ; \hat{\mathbf{D}}_{u}^{(2)} ; \ldots ; \hat{\mathbf{D}}_{u}^{(n)}\right]
$$

with

$$
\hat{\mathbf{D}}_{u}^{(I)}=\left[\begin{array}{ll}
\frac{\partial^{2} N_{I}(\mathbf{X})}{\partial X_{1}^{2}} & 0 \\
\frac{\partial^{2} N_{I}(\mathbf{X})}{\partial X_{1} \partial X_{2}} & 0 \\
0 & \frac{\partial^{2} N_{I}(\mathbf{x})}{\partial X_{1}^{2}} \\
\frac{\partial^{2} N_{I}(\mathbf{x})}{\partial X_{2}^{2}} & 0 \\
0 & \frac{\partial^{2} N_{I}(\mathbf{X})}{\partial X_{1} \partial X_{2}} \\
0 & \frac{\partial^{2} N_{I}(\mathbf{x})}{\partial X_{2}^{2}}
\end{array}\right]
$$

The residual $R_{1}$ is expressed by:

$$
R_{1}=\left[\delta \phi^{e}\right]^{T} \mathbf{r}_{1}
$$

with

$$
\begin{aligned}
& \mathbf{r}_{1}=\int_{V_{0}} \mathbf{B}_{\phi}^{T} \epsilon J \mathbf{C}^{-1} \mathbf{B}_{\phi}\left[\boldsymbol{\phi}^{e}\right]^{k}+\mathbf{B}_{\phi}^{T} J f\left(\epsilon-\epsilon_{0}\right) \mathbf{F}^{-1} \hat{\mathbf{I}} \hat{\mathbf{B}}_{u}\left[\mathbf{u}^{e}\right]^{k} d \Omega \\
& -\int_{V_{0}} \mathbf{B}_{\phi}^{T} \mathbf{F}^{-1} \tilde{\mathbf{P}}^{0}+\mathbf{N}_{\phi}^{T} \tilde{\rho}^{e} d \Omega-\int_{\partial V_{0 D}} \mathbf{N}_{\phi}^{T} D_{n}^{b}, d \Gamma .
\end{aligned}
$$


where the matrix $\tilde{\mathbf{I}}$ is such that $\mathbf{g}=\tilde{\mathbf{I}}[\mathcal{G}(\mathbf{u})]$ and is given by

$$
\hat{\mathbf{I}}=\left[\begin{array}{llllll}
1 & 0 & 0 & 1 & 0 & 0 \\
0 & 0 & 1 & 0 & 0 & 1
\end{array}\right]
$$

\subsection{Case without considering a surrounding medium}

In this case, the residual $\mathbf{r}_{2}$, which is such that

$$
R_{2}=\left[\delta \boldsymbol{\phi}^{e}\right]^{T} \mathbf{r}_{2}
$$

is expressed as:

$$
\begin{aligned}
& \mathbf{r}_{2}=\int_{\Omega_{0}} \overline{\mathbf{B}}_{u}^{T}\left[\mathbf{P}^{e l}\right] d \Omega+\int_{\Omega_{0}} \hat{\mathbf{B}}_{u}^{T}[\mathcal{S}] d \Omega+\int_{\Omega_{0}} \overline{\mathbf{B}}_{u}^{T}\left[\tilde{\boldsymbol{\Sigma}}_{M W}\right] d \Omega \\
& -\int_{\Omega_{0}} \mathbf{N}_{u}^{T} \tilde{\mathbf{f}}^{e} d \Omega-\int_{\partial \Omega_{0 F}} \mathbf{N}_{u}^{T} \tilde{\mathbf{t}}^{e} d \Gamma
\end{aligned}
$$

with

$$
\left[\tilde{\boldsymbol{\Sigma}}_{M W}\right]=-\frac{\epsilon_{0}}{2}|\mathbf{E}|^{2}\left[\mathbf{F}^{-T}\right]-\frac{1}{2 J\left(\epsilon-\epsilon_{0}\right)}|\tilde{\mathbf{P}}|^{2}\left[\mathbf{F}^{-T}\right]+\tilde{\mathbf{Z}}
$$

and

$$
\begin{gathered}
{\left[\mathbf{F}^{-T}\right]=\left[\begin{array}{c}
F_{11}^{-T} \\
F_{12}^{-T} \\
F_{21}^{-T} \\
F_{22}^{-T}
\end{array}\right], \quad[\boldsymbol{\Sigma}]=\left[\begin{array}{c}
\Sigma_{11} \\
\Sigma_{12} \\
\Sigma_{21} \\
\Sigma_{22}
\end{array}\right], \quad\left[\tilde{\boldsymbol{\Sigma}}_{M W}\right]=\left[\begin{array}{l}
\left(\Sigma_{M W}\right)_{11} \\
\left(\Sigma_{M W}\right)_{12} \\
\left(\Sigma_{M W}\right)_{21} \\
\left(\Sigma_{M W}\right)_{22}
\end{array}\right]} \\
{[\mathcal{S}]=\left[\begin{array}{l}
\mathcal{S}_{111} \\
\mathcal{S}_{112} \\
\mathcal{S}_{211} \\
\mathcal{S}_{122} \\
\mathcal{S}_{212} \\
\mathcal{S}_{222}
\end{array}\right], \tilde{\mathbf{Z}}=\left[\begin{array}{l}
E_{1} \tilde{D}_{1} \\
E_{1} \tilde{D}_{2} \\
E_{2} \tilde{D}_{1} \\
E_{2} \tilde{D}_{2}
\end{array}\right]}
\end{gathered}
$$


$|\mathbf{a}|^{2}=\mathbf{a}^{T} \mathbf{a}$ and

$$
\begin{aligned}
& \mathbf{E}=-\mathbf{F}^{-T} \mathbf{B}_{\phi}\left[\boldsymbol{\phi}^{e}\right] \\
& \tilde{\mathbf{P}}=-J\left(\epsilon-\epsilon_{0}\right)\left[f \tilde{\mathbf{I}} \hat{\mathbf{B}}_{u} \mathbf{u}^{e}+\mathbf{F}^{-T} \mathbf{B}_{\phi}\left[\boldsymbol{\phi}^{e}\right]\right] \\
& \tilde{\mathbf{D}}=-J \epsilon \mathbf{C}^{-1} \mathbf{B}_{\phi}\left[\boldsymbol{\phi}^{e}\right]-J f\left(\epsilon-\epsilon_{0}\right) \mathbf{F}^{-1} \hat{\mathbf{I}} \hat{\mathbf{B}}_{u} \mathbf{u}^{e}+\mathbf{F}^{-1} \tilde{\mathbf{P}}_{0} .
\end{aligned}
$$

Above,

$$
[\mathbf{F}]=\left[\begin{array}{l}
1 \\
0 \\
0 \\
1
\end{array}\right]+\mathbf{B}_{u} \mathbf{u}^{e}
$$

\subsection{Case with considering a surrounding medium}

In this case, the residual is expressed as

$$
\begin{aligned}
& \mathbf{r}_{2}=\int_{\Omega_{0}} \overline{\mathbf{B}}_{u}^{T}[\boldsymbol{\Sigma}] d \Omega+\int_{\Omega_{0}} \hat{\mathbf{B}}_{u}^{T}[\mathcal{S}] d \Omega \\
& -\int_{\Omega_{0}} \mathbf{N}_{u}^{T} \tilde{\mathbf{f}}^{e} d \Omega-\int_{\partial \Omega_{0 F}} \mathbf{N}_{u}^{T} \tilde{\mathbf{t}}^{e} d \Gamma-\int_{\partial \Omega_{0}} \mathbf{N}_{u}^{T} \mathbf{f}_{M W}^{S} d \Gamma,
\end{aligned}
$$

where $\mathbf{f}_{M W}^{S}=\left[\left[\tilde{\boldsymbol{\Sigma}}_{M W}\right]\right] \mathbf{N}$. At the interface between the solid dielectric and the surrounding media (e.g. air), external forces are exerted due to the term $\mathbf{f}_{M W}^{s}$ in Eq. (66). In this work, we propose the following simple discretization for the term $\int_{\partial \Omega_{0}} \mathbf{N}_{u}^{T} \mathbf{f}_{M W}^{S} d \Gamma$. Considering an integration point on the interface $\partial \Omega$, we define the approximation of the jump in $\left[\left[\tilde{\boldsymbol{\Sigma}}_{M W}\right]\right] \mathbf{N}$ as:

$$
\left[\left[\tilde{\mathbf{\Sigma}}_{M W}\right]\right] \simeq \tilde{\mathbf{\Sigma}}_{M W}\left(\mathbf{x}^{+}\right)-\tilde{\mathbf{\Sigma}}_{M W}\left(\mathbf{x}^{-}\right)
$$

where $\mathbf{x}^{+}$and $\mathbf{x}^{-}$refer to integration points located in the center of two elements adjescent to the segment containing the point $\mathbf{x}$ (see Fig. 2).

$\frac{\partial u}{\partial x}=0$

It is worth noting that if strain gradient is taken into account, the strong equations in (45) or in (185) are fourth-order nonlinear partial differential equations and require $C^{1}$-continuity of the displacement field and associated $C^{1}$ Finite Elements, while the electric potential field can be discretized with 


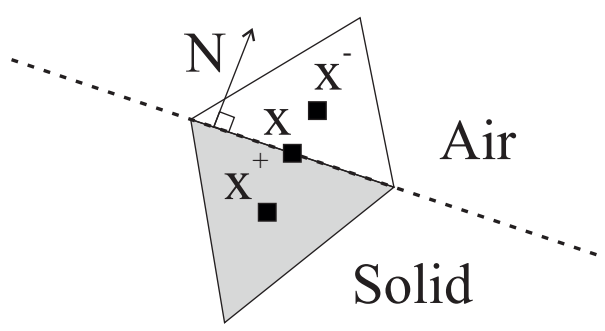

Figure 2. Two adjacent elements across the interface between the solid and surrounding media for the approximation of the Maxwell stress jump across the interface.

$C^{0}$ elements. Even though mixed finite elements requiring only $C^{0}$ continuity could be alternatively considered for handing strain gradient elasticity [34,4], in the present work, we have used linear triangle elements to discretize the potential field, while $C^{1}$ triangular Argyris elements with 21 degrees of freedom per element [11] have been chosen for the displacements. Note that other discretization choices are possible, as meshfree methods [1] or isogeometric FEM [18], which also ensure the $C^{1}$-continuity. The main objective of this paper is to provide the different formulations and linearization procedures, in which the discretization is a numerical ingredient that can be changed. We chose on purpose the simplest $C^{1}$ discretization based on triangular elements for the sake of convenience, but we are aware that such discretization might be cumbersome in 3D. Introducing the mentioned discretization techniques in the present framework is left to further studies.

\subsection{Linearized system}

Introducing approximations (112), (114) and (124) in weak forms (84) and (110) we obtain the following system of linear equations associated with the linearized problem in the Newton algorithm:

$$
\left[\begin{array}{ll}
\mathbf{K}_{11} & \mathbf{K}_{12} \\
\mathbf{K}_{21} & \mathbf{K}_{22}
\end{array}\right]\left[\begin{array}{c}
\Delta \boldsymbol{\phi} \\
\Delta \mathbf{u}
\end{array}\right]=-\left[\begin{array}{l}
\mathbf{r}_{1} \\
\mathbf{r}_{2}
\end{array}\right]
$$

where the different matrices are expressed in the following.

$$
\begin{aligned}
& \mathbf{K}_{11}=\int_{V_{0}} J \epsilon \mathbf{B}_{\phi}^{T} \mathbf{C}^{-1} \mathbf{B}_{\phi} d \Omega \\
& \mathbf{K}_{12}=\int_{\Omega_{0}} \mathbf{B}_{\phi}^{T}\left(\mathbf{M}_{1}+\mathbf{M}_{2}-\mathbf{M}_{4}\right) \overline{\mathbf{B}}_{u}^{T} d \Omega+\int_{\Omega_{0}} \mathbf{B}_{\phi}^{T} \mathbf{M}_{3} \hat{\mathbf{B}}_{u}^{T} d \Omega \\
& \mathbf{K}_{21}=\int_{\Omega_{0}} \hat{\mathbf{B}}_{u}^{T} \mathbf{Q}_{1} \mathbf{B}_{\phi} d \Omega+\int_{\Omega_{0}} \overline{\mathbf{B}}_{u}^{T} \mathbf{Q}_{2} \mathbf{B}_{\phi} d \Omega \\
& \tilde{\mathbf{K}}_{22}=\int_{\Omega_{0}} \overline{\mathbf{B}}_{u}^{T} \mathbf{C}^{e l} \overline{\mathbf{B}}_{u} d \Omega+\int_{\Omega} \hat{\mathbf{B}}_{u}^{T} \mathbf{T} \hat{\mathbf{B}}_{u} d \Omega+\int_{\Omega_{0}} \overline{\mathbf{B}}_{u}^{T}\left(\mathbf{Q}_{3} \overline{\mathbf{B}}_{u}+\mathbf{R} \hat{\mathbf{B}}_{u}\right) d \Omega
\end{aligned}
$$


In the above, $\mathbf{M}_{1}, \mathbf{M}_{2}, \mathbf{M}_{3}, \mathbf{M}_{4}, \mathbf{Q}_{1}, \mathbf{Q}_{2}, \mathbf{C}^{e l}, \mathbf{T}, \mathbf{Q}_{3}$ and $\mathbf{R}$ are the matrix forms corresponding to the operators $\mathcal{M}_{1}, \mathcal{M}_{2}, \mathbb{M}_{3}, \mathcal{M}_{4}, \mathbb{Q}_{1}, \mathbb{Q}_{2}, \mathbb{C}^{e l}, \mathbb{T}, \mathbb{Q}_{3}$ and $\mathbb{R}$, respectively.

Solving the linear system (140), the nodal displacements and electric potentials are udpdated through

$$
\begin{aligned}
& \mathbf{u}^{k+1}=\mathbf{u}^{k}+\Delta \mathbf{u}, \\
& \boldsymbol{\phi}^{k+1}=\boldsymbol{\phi}^{k}+\Delta \boldsymbol{\phi},
\end{aligned}
$$

until a convergence criterion is reached.

\section{A staggered algorithm for simulation of soft solids subjected to Maxwell stress in a surrounding medium}

In this section, we describe a staggered algorithm to solve the full problem of soft dielectrics in surrounding media, subjected to Maxwell stress. Due to severe nonlinearities, solving the problem (140) in a monolithical fashion may lead to convergence issues. To alleviate this problem, the following algorithm can be employed. To avoid spurious distortions of the mesh related to the surrounding medium when the domain $\Omega$ is deformed, a displacement field in the surrounding domain is artificially defined, and fictious elastic properties are assigned to the surrounding domain defined in $\bar{\Omega}_{0}$. Let $\phi^{n}(\mathbf{x}), \mathbf{u}^{n}(\mathbf{x})$ $\mathbf{v}^{n}(\mathbf{x})$ denote the potential field in $V_{0}$, the displacement field in $\Omega_{0}$ and the displacement field in $\bar{\Omega}_{0}$ at time step $t^{n}$. The algorithm runs as follows:

(1) For each time step $t^{n+1}$ (increment of external electric or mechanical loading)

(a) Given $\mathbf{u}^{n}$, solve the dielectric problem (54) $\forall \mathbf{x} \in V_{0}$, to find $\phi^{n+1}(\mathbf{x})$.

(b) Compute the surface Maxwell forces $\mathbf{f}_{M W}^{S}\left(\phi^{n+1}, \mathbf{u}^{n}\right)=\left[\left[\tilde{\mathbf{\Sigma}}_{M W}\left(\phi^{n}, \mathbf{u}^{n}\right)\right]\right] \mathbf{N}$, using (38), (39) and the discretization procedure described in section 6.

(c) Solve the mechanical problem (66) $\forall \mathbf{x} \in \Omega_{0}$, to find $\mathbf{u}^{n+1}(\mathbf{x})$.

(d) Prescribe Dirichlet boundary conditions obtained from (c) on $\partial \Omega_{0}$ in $\bar{\Omega}_{0}$ using $\mathbf{u}^{n+1}(\mathbf{x})$ and solve the fictious elastic problem in $\bar{\Omega}_{0}$ to find $\mathbf{v}^{n+1}(\mathbf{x})$

(e) Update

- $\phi^{n}(\mathbf{x})=\phi^{n+1}(\mathbf{x})$

- $\mathbf{u}^{n+1}(\mathbf{x})=\mathbf{u}^{n}(\mathbf{x})$

- $\mathbf{v}^{n+1}(\mathbf{x})=\mathbf{v}^{n}(\mathbf{x})$

(2) Go to (1) 


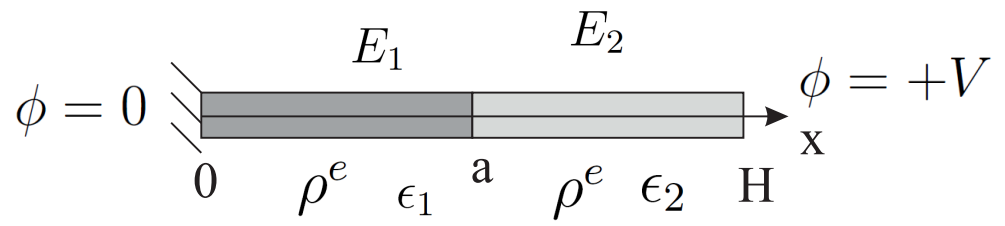

Figure 3. Bilayer material with uniform charge $\rho$ subjected to voltage.

Note that this algorithm uses the Newton method described in the previous sections, but only avoids the linearization of the surface term by prescribing it as a given force term known from the previous iteration.

\section{Applications: numerical examples}

\subsection{Effective piezoelectricity induced by coupling electrets and Maxwell stress}

The objective of this first example is to validate the present framework in the context of Maxwell stress at small strains. A unidimensional structure is composed of two layers with respective Young's modulus $E_{1}$ and $E_{2}$ and permittivity coefficients $\epsilon_{1}$ and $\epsilon_{2}$, as depicted in Figure 3.

A charge $\rho^{e}(x)$ is uniformly distributed over the structure. The $(x=0)$-side is subjected to a potential $\phi=0$ and clamped, while the $(x=H)$-side is subjected to a potential $\phi=+V$ and is free of mechanical loading. In 1D, we have:

$$
e(x)=\frac{d \phi(x)}{d x}, \quad D(x)=-\epsilon \frac{d \phi(x)}{d x}, \quad p(x)=-\left(\epsilon-\epsilon_{0}\right) \frac{d \phi(x)}{d x}
$$

where $\phi, e, D$, and $p$ denote electric potential, electric field, electric displacement and polarization, respectively. Then the equations of the dielectric problem degenerate to:

$$
\frac{d D(x)}{d x}=\rho^{e}
$$

or

$$
\frac{d^{2} \phi(x)}{d x}=-\frac{\rho^{e}}{\epsilon}
$$

with continuity equations at the interface:

$$
[[\phi(a)]]=0, \quad[[D(a)]]=0 .
$$


Then the solution $\phi(x)$ in each domain is given in the form:

$$
\phi(y)=\left\{\begin{array}{l}
-\frac{\rho^{e} x^{2}}{2 \epsilon_{2}}+A_{1} x, \quad 0 \leq x \leq a \\
\frac{\rho^{e} x^{2}}{2 \epsilon_{1}}+A_{2} x+A_{3}, \quad a \leq x \leq H
\end{array}\right.
$$

where $A_{1}, A_{2}$ and $A_{3}$ are solution of:

$$
\left[\begin{array}{rrr}
0 & H & 1 \\
a & -a & 1 \\
\epsilon_{2} & \epsilon_{1} & 0
\end{array}\right]\left[\begin{array}{l}
A_{1} \\
A_{2} \\
A_{3}
\end{array}\right]=\left[\begin{array}{l}
V+\frac{\rho^{e} H^{2}}{2 \epsilon_{1}} \\
-\frac{a^{2} \rho^{e}}{2}\left(\frac{1}{\epsilon_{1}}-\frac{1}{\epsilon_{2}}\right) \\
0
\end{array}\right] .
$$

The first line of the system is associated with the boundary condition $\phi(H)=$ $V$, the second one with the continuity of potential across the interface $(x=a)$, and the last one with the continuity of electric displacement at the interface.

Then we note that the mechanical problem can be solved in an uncoupled manner, given the solution $\phi(x)$. In $1 \mathrm{D}$, the equation of the mechanical balance problem is given by:

$$
\frac{d\left(\sigma^{e}(x)+\tilde{\sigma}_{M W}(x)\right)}{d x}=0
$$

with

$$
\begin{aligned}
& \sigma^{e}(x)=E \frac{d u(x)}{d x} \\
& \tilde{\sigma}_{M W}(x)=-\frac{\epsilon_{0}}{2}\left(\frac{d \phi(x)}{d x}\right)^{2}+e(x) d(x)-\frac{\epsilon-\epsilon_{0}}{2}\left(\frac{d \phi(x)}{d x}\right)^{2} \\
& =\frac{\epsilon}{2}\left(\frac{d \phi(x)}{d x}\right)^{2} .
\end{aligned}
$$

Then Eq. (153) can be rewritten as:

$$
E \frac{d u(x)}{d x}=-\epsilon \frac{d^{2} \phi(x)}{d x^{2}} \frac{d \phi(x)}{d x}
$$

with continuity equations at the interface:

$$
[[u(a)]]=0, \quad\left[\left[\sigma^{e}(a)+\tilde{\sigma}_{M W}(a)\right]\right]=0
$$

and boundary conditions

$$
u(0)=0, \quad \sigma^{e}(H)+\sigma_{M W}(H)=0 .
$$


Then solving the displacement equation leads to

$$
u_{y}(y)=\left\{\begin{array}{l}
\frac{\rho^{e}}{E_{2}}\left(B_{2} x+\frac{A_{1} x^{2}}{2}-\frac{\rho^{e} x^{3}}{6 \epsilon_{2}}\right), \quad 0 \leq x \leq a \\
\frac{\rho^{e}}{E_{1}}\left(B_{3}+B_{1} x+\frac{A_{2} x^{2}}{2}-\frac{\rho^{e} x^{3}}{6 \epsilon_{1}}\right), \quad a \leq x \leq H
\end{array}\right.
$$

where $B_{1}, B_{2}$ and $B_{3}$ are solutions of:

$$
\left[\begin{array}{ccc}
\rho^{e} & 0 & 0 \\
1 & -1 & 0 \\
\frac{a}{E_{1}} & -\frac{a}{E_{2}} & \frac{1}{E_{1}}
\end{array}\right]\left[\begin{array}{c}
B_{1} \\
B_{2} \\
B_{3}
\end{array}\right]=\left[\begin{array}{c}
F_{1} \\
F_{2} \\
F_{3}
\end{array}\right] .
$$

In Eq. (160), the first equation corresponds to the free load condition at ( $x=$ $H$ ), the second one to the continuity of total stress across the interface, and the last one to the continuity of displacements across the interface, and

$$
\begin{aligned}
& F_{1}=\rho^{e}\left(\frac{\rho^{e} H^{2}}{2 \epsilon_{1}}-A_{2} H\right)-\frac{\epsilon_{1}}{2}\left(\frac{\rho^{e} H}{\epsilon_{1}} A_{2}\right)^{2} \\
& F_{2}=\frac{\rho^{e} a^{2}}{2}\left(\frac{1}{\epsilon_{1}}-\frac{1}{\epsilon_{2}}\right)+a\left(A_{1}-A_{2}\right)+\frac{\epsilon_{2}}{2 \rho^{e}}\left(A_{1}-\frac{\rho^{e} a}{\epsilon_{2}}\right)^{2} \\
& F_{3}=\frac{1}{E_{2}}\left(\frac{A_{1} a^{2}}{2}-\frac{\rho^{e} a^{3}}{6 \epsilon_{2}}\right)+\frac{1}{E_{1}}\left(\frac{\rho^{e} a^{3}}{6 \epsilon_{1}}-\frac{A_{2} a^{2}}{2}\right) .
\end{aligned}
$$

The effective piezoelectric coefficient is computed as

$$
d^{e f f}=\frac{\partial u_{y}(H)}{\partial V} .
$$

Comparisons between FEM simulation and the exact solution are presented in Figure 4 for different $E_{2} / E_{1}$ ratio, with $\rho^{e}=0.001 \mathrm{C} / \mathrm{m}^{3}, E_{1}=1 \mathrm{MPa}$, $\epsilon_{1}=2 \epsilon_{0}, \epsilon_{2}=20 \epsilon_{0}, H=1 \mathrm{~mm}, a=H / 2$. The results show a very good agreement between the theory and the numerical soltuon, which validates the present numerical framework in this case.

\subsection{Flexoelectricity}

In this second example, flexoelectricity is considered, i.e. the material parameters $f$ and $g$ in Eq. (24) are no more neglected. We neglect Maxwell stress and the small strain assumption is adopted. A uniform bar is submitted to the conditions described in Fig. 5. 


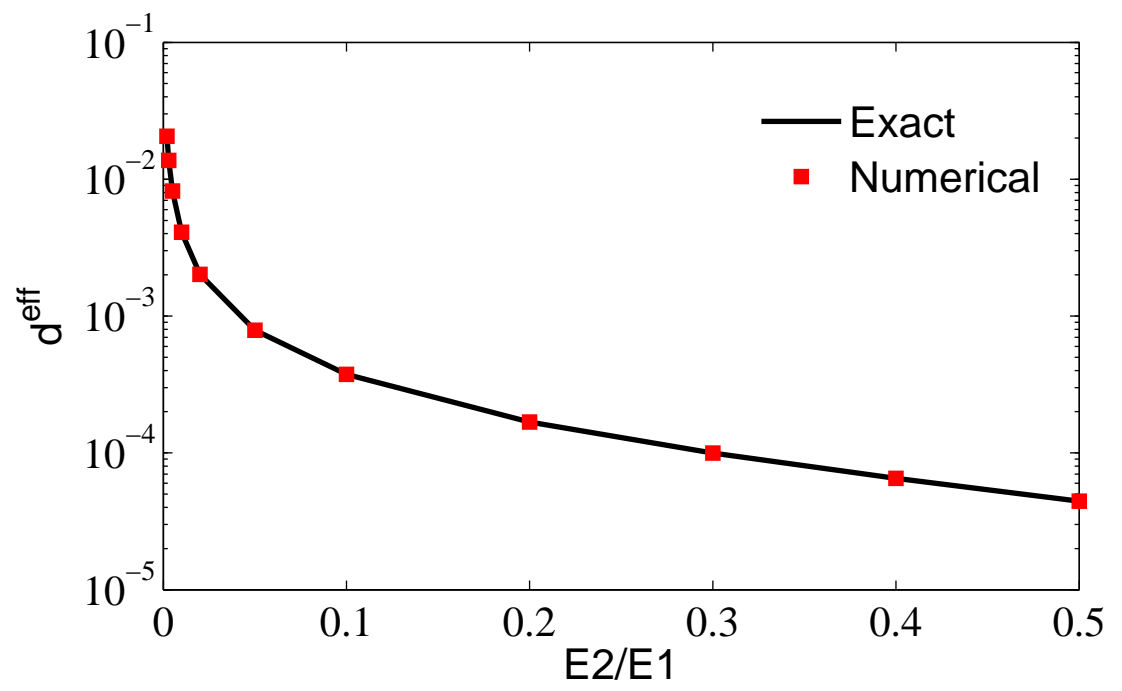

Figure 4. Effective piezoelectric coefficient $d^{e f f}$ as a function of the ratio $E_{2} / E_{1}$.

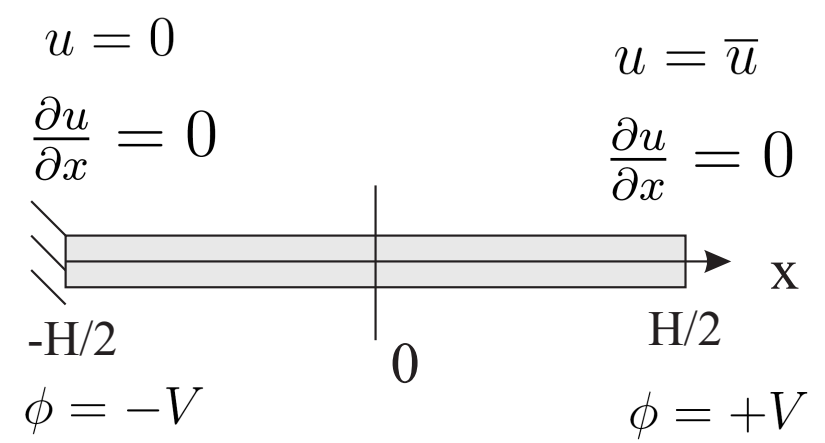

Figure 5. Layer with flexoelectric properties subjected to voltage.

The material parameters are chosen as follows: $\epsilon_{0}=8.854 \mathrm{C} / \mathrm{m}^{2}, \epsilon=17.708$ $\mathrm{C} / \mathrm{m}^{2}, E=1 \mathrm{MPa}, \nu=0$, the elastic coefficient $g$ associated to strain gradient rigidity in (24) varies between $10^{-3}$ and 1 , the flexoelectric coefficient $f$ in (24) varies between 0 and $10^{-1}, \rho^{e}=0 \mathrm{C} / \mathrm{m}^{3}, \mathbf{p}^{0}=\mathbf{0} \mathrm{V} / \mathrm{m}, \bar{u}=0.1 \mathrm{~mm}, V=10^{-3}$ $\mathrm{V}, H=1 \mathrm{~mm}$. The analytical solution of this problem is given by:

$$
\begin{aligned}
u(x) & =\bar{u} e^{\frac{-x}{k}} \frac{-2 k e^{\frac{H}{2 k}}+2 k e^{\frac{H+4 x}{2 k}}+e^{\frac{H+x}{k}}(2 k-L-2 x)-e^{\frac{x}{k}}(2 k+L+2 x)}{2\left(2 k\left[e^{\frac{H}{k}}-1\right]-H\left[e^{\frac{H}{k}}+1\right]\right)} \\
\phi(x) & =\frac{\bar{u} G_{u}+V G_{V}}{\epsilon H\left(2 k-2 k e^{\frac{H}{k}}+H+H e^{\frac{H}{k}}\right)}
\end{aligned}
$$

with

$$
G_{u}=-e^{\frac{-x}{k}}\left\{-e^{\frac{H}{2 k}}+e^{\frac{x}{k}}+e^{\frac{L+x}{k}}-e^{\frac{L+4 x}{2 k}}\right\}\left(\epsilon-\epsilon_{0}\right) f H,
$$




$$
G_{V}=-2 x \epsilon\left(2 k\left[e^{\frac{H}{k}}-1\right]-H\left[e^{\frac{H}{k}}+1\right]\right) .
$$

In the above, $k=\sqrt{A / E}$ and

$$
A=g-\left(\epsilon-\epsilon_{0}\right) f^{2}\left[1-\frac{\left(\epsilon-\epsilon_{0}\right)}{\epsilon}\right]
$$

To our knowledge, this solution is provided in this paper for the first time.

To solve numerically the problem, we model the one-dimensional problem in a two-dimensional domain by prescribing appropriate boundary conditions to render the problem 1D. A square domain has been discretized into $15 \times$ 15 nodes, corresponding to 392 regular triangular Argyris [11] elements to guarantee the $C^{1}$-continuity.

In Figs. 6-9 the displacement solution as well as the potential distribution along $x$ is provided. First, the elasticity gradient coefficient $g$ has been varied for a fixed value of the flexoelectric coefficient $f=0.01$. Second, $f$ has been varied for a fixed value of $g=1$. In each case, the exact solution has been compared with the numerical solution. We can note here again a very good agreement between the analytical and numerical solutions.

\subsection{Finite strains: soft cylindrical body in an electrical field}

In this example, we consider a soft cylindrical body subjected to an electric field in a finite square box, as described in Fig. 8. Related experimental and numerical works can be found e.g. in [37,30,22]. The geometry and boundary conditions of the problem are depicted in Fig. 8 (a), and the corresponding mesh is depicted in Fig. 8 (b). For the sake of symmetry, only a half of the problem is modeled and meshed. The body is surrounded by air in the square domain. A voltage is applied on the upper $(y=-H)$ and lower $(y=H)$ ends of the square domain. For the sake of simplicity, plane strain are assumed. The material properties of the soft body are: $E=0.01 \mathrm{MPa}, \nu=0.45$. The staggered algorithm described in section 7 has been used.

Due to the Maxwell stress, the body is stretched when the voltage is increased, as depicted in Fig. 9. It is worth noting that due to the surface term $\left[\left[\tilde{\boldsymbol{\Sigma}}_{M W}\right]\right] \mathbf{N}$ in (66), the initial circular section does not remain an ellipsoid but takes a more complex shape.

The evolution of the stretch $\lambda_{1}$ in the $y$-direction as a function of the applied electric current $\left|\mathbf{E}_{0}\right|^{2}, \mathbf{E}_{0}=2 V \mathbf{e}_{2}$ is depicted in Fig. 10 for several values of the soft body's relative permittivity $\epsilon^{r}=\epsilon / \epsilon_{0}$. 


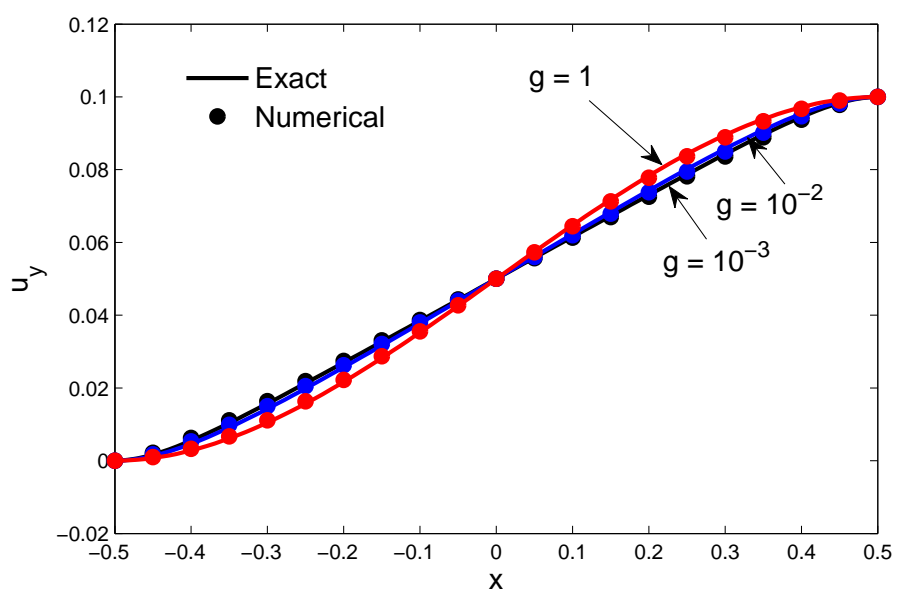

(a)

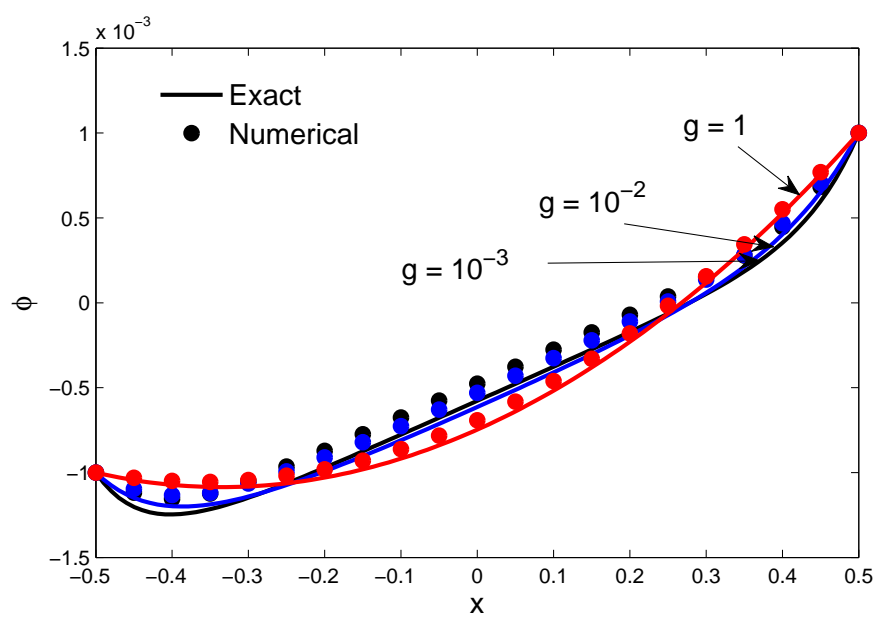

(b)

Figure 6. Solution field along the layer thickness for a fixed flexoelectric coefficient $f=0.01$ and a varying strain gradient coefficient $g$; (a) Displacement; (b) electric potential.

Due to severe nonlinearities and probably the occurrence of instabilities, the present formulation was not able to simulate larger strains than the ones indicated in Fig. 10. Implementation of more robust algorithms for taking into account these nonlinearities and possible instabilities are reported to future studies, e.g. by implementing an arc-length control algorithm, or by introducing inertia effects, as proposed by Park et al. in [29].

\section{Conclusion}

In the present work, a finite element framework has been introduced to solve the response of nonlinear, flexoelectric soft dielectric materials at small and 


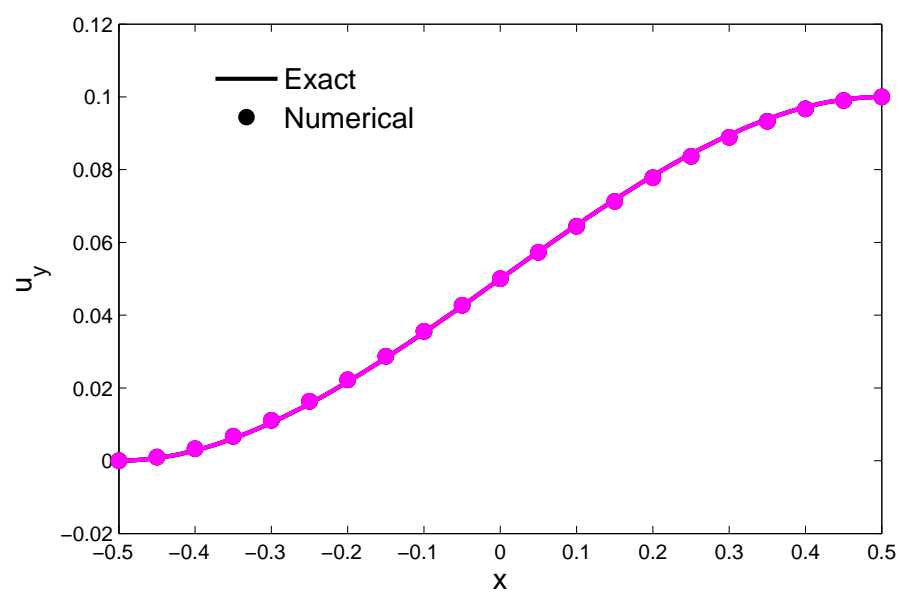

(a)

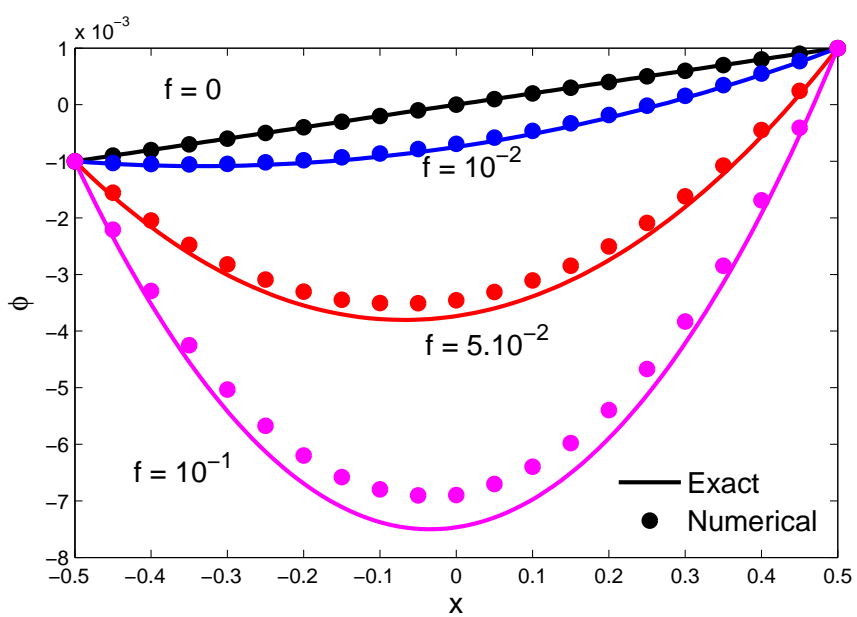

(b)

Figure 7. Solution field along the layer thickness for a fixed strain gradient coefficient $g=1$ and a varying flexoelectric coefficient ; (a) Displacement; (b) electric potential.

finite strains, with effects of Mawxell stress. Weak forms and finite element discretizations able to handle strain gradient in the context of flexoelectricity have been introduced. Numerical algorithms for the treatment of a soft dielectric in a surrounding medium have been proposed, more specifically to handle the effects of discontinuities of the Maxwell stress at the interfaces. Finally, several benchmarks have been presented to assess the present formulations and numerical schemes, through applications of special cases of interest: induced piezoelectricity in non-piezoelectric materials due to coupling of Maxwell stress and electrets, flexoelectricity, or stretching of electroactive soft dielectrics and polymers subjected to an external electric field. 


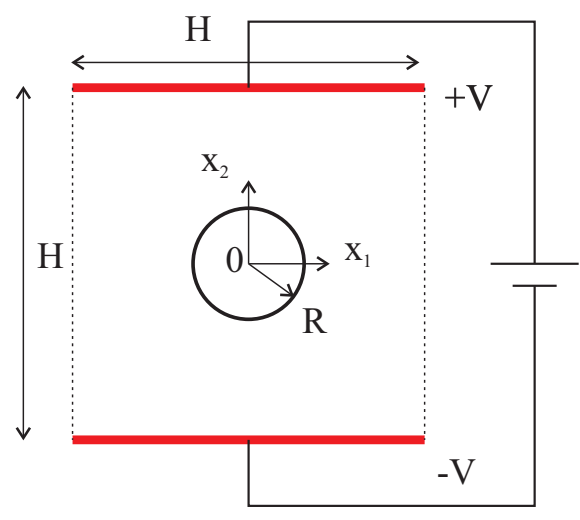

(a)

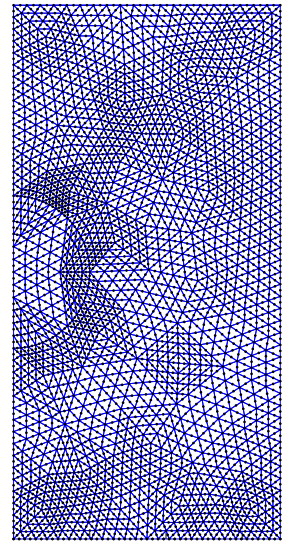

(b)

Figure 8. Soft cylindrical body subjected to an electric field; (a) geometry; (b) FEM mesh.

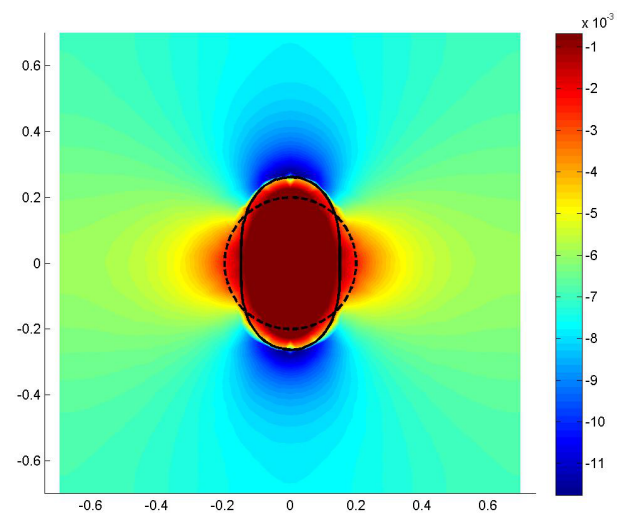

(a)

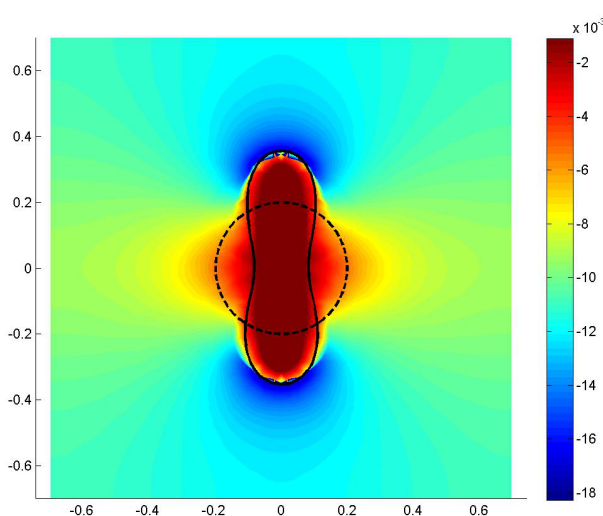

(b)

Figure 9. Electric field $\tilde{E}(\mathbf{x})_{2}$ and deformed configuration of the ellipsoid (magnified 10 times) for an applied electric field (a) $E_{0}=0.009 \mathrm{~V} / \mathrm{m}$ and (b) $E_{0}=0.141 \mathrm{~V} / \mathrm{m}$, $\epsilon_{r}=20, E=0.01 \mathrm{MPa}, \nu=0.45$.

\section{Aknowledgements}

Fruitful discussions with Prof. Pradeep Sharma, The University of Houston, are gratefuly aknowledged. J.Y. thanks the financial support of Institut Universitaire de France (IUF). L.L. gratefully acknowledges the support of NSF CMMI-135156, DMS-1410273, AFOSR-FA9550-16-1-0181 and NSFC1152800009 .

\section{Appendix: Particular cases}

Here, we particularize the above developments to special cases of interests. 


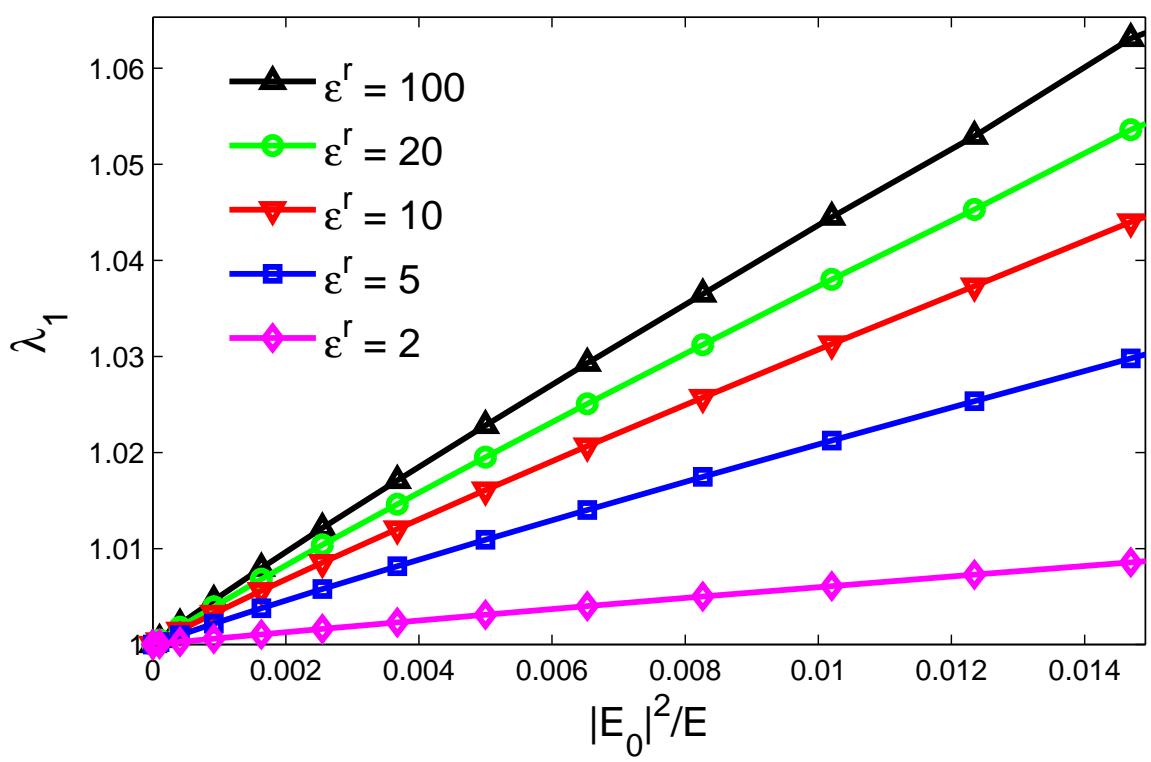

Figure 10. Stretch of the initial spherical particle as a function of the applied electric current $\left|\mathbf{E}_{0}\right|^{2}$.

\subsection{Flexoelectric elastomers at finite strains}

If Maxwell stress is neglected but the material is flexoelectric, we obtain the following equations for finite strains flexoelectricity (we assume that we do not consider the surrounding medium here):

\subsubsection{Strong form}

$$
\nabla_{X} \cdot(\tilde{\mathbf{D}})=\tilde{\rho}^{e} \quad \text { in } V_{0}
$$

where $\tilde{\mathbf{D}}$ is given by (33), with boundary conditions

$$
\begin{aligned}
& \phi=\phi^{b} \text { on } \partial V_{0 \phi}, \\
& \mathbf{N} \cdot \tilde{\mathbf{D}}=D_{n}^{b} \text { on } \partial V_{D} . \\
& \nabla_{X} \cdot \boldsymbol{\Sigma}-\nabla_{X} \cdot\left(\nabla_{X} \cdot \mathcal{S}\right)+\tilde{\mathbf{f}}^{e}=0 \text { in } \Omega_{0},
\end{aligned}
$$

with boundary conditions

$$
\mathbf{u}=\mathbf{u}^{b} \text { on } \partial \Omega_{u 0},
$$

$$
\boldsymbol{\Sigma} \mathbf{N}-\left(\nabla_{X} \cdot \mathcal{S}\right) \mathbf{N}-\tilde{\mathbf{t}}^{e}=0 \text { on } \partial \Omega_{F 0},
$$

and

$$
\mathcal{S N} \otimes \mathbf{N}=0 \text { on } \partial \Omega_{0} .
$$




\subsubsection{Weak form}

The weak form is straightforwardly expressed by

(54) and

$$
\begin{aligned}
& \int_{\Omega_{0}}(\boldsymbol{\Sigma}: \delta \mathbf{F}+\mathcal{S} \vdots \delta \mathcal{G}) d \Omega-\int_{\Omega_{0}} \tilde{\mathbf{f}}^{e} \cdot \delta \mathbf{u} d \Omega-\int_{\partial \Omega_{0 F}} \tilde{\mathbf{t}}^{e} d \Gamma \\
& =-R_{2}(\mathbf{u}, \phi)=0 .
\end{aligned}
$$

\subsubsection{Consistent linearization}

The linearized problem of finite strain flexoelectricity is given by:

(84) and

$$
\begin{aligned}
& \int_{\Omega_{0}} \mathcal{G}(\delta \mathbf{u}) \vdots \mathbb{Q}_{1} \cdot \nabla_{X}(\Delta \phi) d \Omega+\int_{\Omega_{0}} \delta \mathbf{F}: \mathbb{C}^{e l}: \Delta \mathbf{F} d \Omega \\
& +\int_{\Omega_{0}} \mathcal{G}(\delta \mathbf{u}) \vdots \mathbb{T} \vdots \Delta \mathcal{G} d \Omega=-R_{2}\left(\mathbf{u}^{k}, \phi^{k}\right) .
\end{aligned}
$$

\subsection{Dielectric elastomers without flexoelectricity and Maxwell stress}

The case of taking into account Maxwell stress in a non-flexoelectric material is simply obtained by taking $f=0$ and $g=0$ in the formulations of sections 3 and 4.

\subsection{Assumption of small strains}

In this section, we consider the case where small perturbations are assumed. In this case, $J \simeq 1, \mathbf{C} \simeq \mathbf{1}, \mathbf{F} \simeq \mathbf{1}$.

The strong form is given as follows: 
For the dielectric problem:

$$
\nabla \cdot(\mathbf{d})=\rho^{e} \text { in } V
$$

with $\mathbf{e}=-\nabla \phi$,

$$
\begin{aligned}
& \mathbf{d}=-\epsilon \nabla \phi-\left(\epsilon-\epsilon_{0}\right) f \mathbf{g}+\mathbf{p}^{0}, \\
& \mathbf{p}=-\left(\epsilon-\epsilon_{0}\right)[f \mathbf{g}+\nabla \phi] .
\end{aligned}
$$

where $\mathbf{p}^{0}$ is the extrinsic polarization and

$$
g_{i}=\nabla \varepsilon_{i k k}
$$

with boundary conditions

$$
\begin{aligned}
& \phi=\phi^{b} \text { on } \partial V_{\phi} \\
& \mathbf{n} \cdot \mathbf{d}=d_{n}^{b} \text { on } \partial V_{D} .
\end{aligned}
$$

For the mechanical problem:

$$
\nabla \cdot \boldsymbol{\sigma}-\nabla \cdot\left(\nabla \cdot \mathcal{S}^{\prime}\right)+\nabla \cdot \tilde{\boldsymbol{\sigma}}_{M W}+\mathbf{f}^{e}=0 \text { in } \Omega
$$

with boundary conditions

$$
\begin{aligned}
& \mathbf{u}=\mathbf{u}^{b} \text { on } \partial \Omega_{u}, \\
& \boldsymbol{\sigma} \mathbf{n}-\left(\nabla \cdot \mathcal{S}^{\prime}\right) \mathbf{n}+\left[\left[\tilde{\boldsymbol{\sigma}}_{M W}\right]\right] \mathbf{n}-\mathbf{t}^{e}=0 \text { on } \partial \Omega_{F}
\end{aligned}
$$

and

$$
\mathcal{S}^{\prime}: \mathbf{n} \otimes \mathbf{n}=0 \quad \text { on } \partial \Omega
$$

with

$$
\tilde{\boldsymbol{\sigma}}_{M W}=-\frac{\epsilon_{0}}{2}|\mathbf{e}|^{2} \mathbf{1}-\frac{|\mathbf{p}|^{2}}{2\left(\epsilon-\epsilon_{0}\right)}+\mathbf{e} \otimes \mathbf{d}
$$

With definition (24), we obtain, after algebraic computations:

$$
\left(\mathcal{S}^{\prime}\right)_{i j k}=\frac{\partial \psi^{\text {flexo }}}{\partial \nabla \varepsilon_{i j k}}=\frac{g}{2}\left(\nabla \varepsilon_{i p p} \delta_{j k}+\delta_{i k} \nabla \varepsilon_{j p p}\right)+\frac{f}{2}\left(p_{i} \delta_{j k}+\delta_{i k} p_{j}\right)
$$

The weak form associated with the dielectric problem is given by

$$
\begin{aligned}
& \int_{V} \epsilon \nabla \phi \cdot \nabla(\delta \phi) d \Omega+\int_{\Omega}\left(\epsilon-\epsilon_{0}\right) f \mathbf{g} \cdot \nabla(\delta \phi) d \Omega \\
& -\int_{V} \mathbf{p}^{0} \cdot \nabla(\delta \phi) d \Omega-\int_{V} \rho^{e} \delta \phi d \Omega=R_{1}\left(\mathbf{u}^{k}, \phi^{k}\right)
\end{aligned}
$$

The weak form associated with the mechanical problem is given as follows.

Let us define $\left(\mathcal{G}^{\prime}\right)_{i j k}=\frac{\partial u_{i}}{\partial x_{j} \partial x_{k}}$. As $\left(\mathcal{S}^{\prime}\right)_{i j k}$ is symmetric with respect to $i$ and $j$ 
indices, and as $\nabla \varepsilon_{i j k}=\nabla \varepsilon_{j i k}$, we have:

$$
\mathcal{S}_{i j k}^{\prime} \mathcal{G}_{i j k}^{\prime}=\mathcal{S}_{i j k}^{\prime} \frac{1}{2}\left(\mathcal{G}_{j i k}^{\prime}+\mathcal{G}_{i j k}^{\prime}\right)=\mathcal{S}_{i j k}^{\prime} \nabla \varepsilon_{i j k}
$$

We finally obtain the weak form for the mechanical problem:

$$
\begin{aligned}
& \int_{\Omega} \boldsymbol{\sigma}: \boldsymbol{\varepsilon}(\delta \mathbf{u}) d \Omega+\mathcal{S}^{\prime}: \nabla \varepsilon(\delta \mathbf{u}) d \Omega+\int_{\Omega} \tilde{\boldsymbol{\sigma}}_{M W}: \boldsymbol{\varepsilon}(\delta \mathbf{u}) d \Omega \\
& -\int_{\Omega} \mathbf{f}^{e} \cdot \delta \mathbf{u} d \Omega-\int_{\partial \Omega_{F}} \mathbf{t}^{e} \cdot \delta \mathbf{u} d \Gamma=0
\end{aligned}
$$

where $\nabla \varepsilon(\delta \mathbf{u})_{i j k}=\frac{\partial \delta u_{i}}{\partial x_{j} \partial x_{k}}$. We notice that even considering small perturbations, the problem remains nonlinear due to the Maxwell stress term. Then, a linearization of the problem around a know solution $\left(\phi^{k}, \mathbf{u}^{k}\right)$ is performed through:

$$
\begin{aligned}
& R_{1}^{\prime}\left(\mathbf{u}^{k}+\Delta \mathbf{u}, \phi^{k}+\Delta \phi\right) \simeq \\
& R_{1}^{\prime}\left(\mathbf{u}^{k}, \phi^{k}\right)+D_{\Delta \mathbf{u}} R_{1}^{\prime}\left(\mathbf{u}^{k}, \phi^{k}\right)+D_{\Delta \phi} R_{1}^{\prime}\left(\mathbf{u}^{k}, \phi^{k}\right) . \\
& R_{2}^{\prime}\left(\mathbf{u}^{k}+\Delta \mathbf{u}, \phi^{k}+\Delta \phi\right) \simeq \\
& R_{2}^{\prime}\left(\mathbf{u}^{k}, \phi^{k}\right)+D_{\Delta \mathbf{u}} R_{2}^{\prime}\left(\mathbf{u}^{k}, \phi^{k}\right)+D_{\Delta \phi} R_{2}^{\prime}\left(\mathbf{u}^{k}, \phi^{k}\right) .
\end{aligned}
$$

In the following, we develop the different terms associated with the above linearization process. We can express:

$$
D_{\Delta \phi}\left\{\left(\epsilon-\epsilon_{0}\right) f \mathbf{g} \cdot \nabla(\delta \phi)\right\}=\nabla(\delta \phi) \cdot \hat{\mathbb{M}} \vdots \nabla \boldsymbol{\varepsilon}(\Delta \mathbf{u})
$$

with

$$
(\hat{\mathbb{M}})_{i j k l}=\frac{f\left(\epsilon-\epsilon_{0}\right)}{2}\left(\delta_{i j} \delta_{k l}+\delta_{i k} \delta_{j l}\right) .
$$

Then, the linearized form associated with the dielectric problem is obtained as:

$$
\int_{V} \epsilon \nabla(\Delta \phi) \cdot \nabla(\delta \phi) d \Omega+\int_{V} \nabla(\delta \phi) \cdot \hat{\mathbb{M}} \vdots \nabla \varepsilon(\Delta \mathbf{u}) d \Omega=-R_{1}^{\prime}\left(\mathbf{u}^{k}, \phi^{k}\right)
$$

To derive the linearized equations associated with the mechanical problem, we 
note that:

$$
\frac{\partial \mathcal{S}_{i j k}^{\prime}}{\partial \nabla \phi_{l}}=-\frac{f\left(\epsilon-\epsilon_{0}\right)}{2}\left(\delta_{i l} \delta_{j k}+\delta_{i k} \delta_{j l}\right)=-(\hat{\mathbb{M}})_{i l k j} .
$$

We have

$$
\frac{\partial \boldsymbol{\sigma}_{M W}}{\partial \nabla \phi}=\hat{\mathcal{Q}}_{3}^{A}+\hat{\mathcal{Q}}_{3}^{B}+\hat{\mathcal{Q}}_{3}^{C}
$$

with

$$
\begin{aligned}
& \left(\hat{\mathcal{Q}_{3}^{A}}\right)_{i j k}=-\epsilon_{0} \delta_{i j} e_{k}, \\
& \left(\hat{\mathcal{Q}_{3}^{B}}\right)_{i j k}=-\left(\delta_{i k} d_{j}+\epsilon e_{i} \delta_{j k}\right), \\
& \left(\hat{\mathcal{Q}_{3}^{C}}\right)_{i j k}=\delta_{i j} p_{k}
\end{aligned}
$$

We set:

$$
\frac{\partial \tilde{\boldsymbol{\sigma}}_{M W}}{\partial \nabla \boldsymbol{\epsilon}}=\hat{\mathbb{R}}^{A}+\hat{\mathbb{R}}^{B}
$$

with

$$
\left(\hat{\mathbb{R}}^{A}\right)_{i j k l p}=-\frac{e_{i}\left(\epsilon-\epsilon_{0}\right)}{2} f\left[\delta_{j k} \delta_{l p}+\delta_{j l} \delta_{k p}\right]
$$

and

$$
\left(\hat{\mathbb{R}^{B}}\right)_{i j k l p}=\frac{f}{2} \delta_{i j}\left[\delta_{l p} p_{k}+\delta_{k p} p_{l}\right] .
$$

Finally

$$
D_{\Delta \mathbf{u}}\left\{\mathcal{S}^{\prime} \vdots \nabla \varepsilon(\delta \mathbf{u})\right\}=\nabla \varepsilon(\delta \mathbf{u}) \vdots \hat{\mathbb{T}} \vdots \nabla \varepsilon(\Delta \mathbf{u}) .
$$

with

$$
\begin{aligned}
& \hat{\mathbb{T}}=\frac{\partial \mathcal{S}^{\prime}}{\partial \nabla \varepsilon}, \\
& (\hat{\mathbb{T}})_{i j k l m p}=\frac{g}{4}\left(\delta_{i k}\left[\delta_{j l} \delta_{m p}+\delta_{j m} \delta_{l p}\right]+\delta_{j k}\left[\delta_{i l} \delta_{m p}+\delta_{i m} \delta_{l p}\right]\right) .
\end{aligned}
$$

After other similar computations (see section 5), we finally obtain the linearized form associated with the mechanical problem in the case of small strains assumption: 


$$
\begin{aligned}
& \quad-\int_{\Omega} \nabla \boldsymbol{\varepsilon}(\delta \mathbf{u}) \vdots \mathbb{M}^{T} \cdot \nabla(\Delta \phi) d \Omega+\int_{\Omega} \delta \boldsymbol{\varepsilon}: \hat{\mathcal{Q}}_{3} \cdot \nabla(\Delta \phi) d \Omega \\
& \quad+\int_{\Omega} \boldsymbol{\varepsilon}(\Delta \mathbf{u}): \mathbb{C}: \boldsymbol{\varepsilon}(\delta \mathbf{u}) d \Omega+\int_{\Omega} \nabla \boldsymbol{\varepsilon}(\delta \mathbf{u}) \vdots \hat{\mathbb{T}}: \nabla \boldsymbol{\varepsilon}(\Delta \mathbf{u}) d \Omega \\
& \quad+\int_{\Omega} \boldsymbol{\varepsilon}(\delta \mathbf{u}): \hat{\mathbb{R}} \vdots \nabla \boldsymbol{\varepsilon}(\Delta \mathbf{u}) d \Omega=-R_{2}^{\prime}\left(\mathbf{u}^{k}, \phi^{k}\right), \\
& \text { with } \hat{\mathbb{Q}}_{3}=\hat{\mathbb{Q}}_{3}^{A}+\hat{\mathbb{Q}}_{3}^{B}, \hat{\mathbb{R}}=\hat{\mathbb{R}}^{A}+\hat{\mathbb{R}}^{B} .
\end{aligned}
$$

\section{Appendix: discretization for small strains}

In this appendix, we consider case of small strains assumption, taking into account flexoelectricity and Maxwell stress. The electric potential increment and associated test function are expressed in the same manner than in the previous section.

Next, we classically introduce the vector $[\Delta \varepsilon]$ associated with the tensor $\Delta \varepsilon$ :

$$
[\Delta \boldsymbol{\varepsilon}]=\left[\begin{array}{l}
\varepsilon_{11}(\Delta \mathbf{u}) \\
\varepsilon_{22}(\Delta \mathbf{u}) \\
2 \varepsilon_{12}(\Delta \mathbf{u})
\end{array}\right]=\left[\begin{array}{l}
\frac{\partial \Delta u_{1}}{\partial x_{1}} \\
\frac{\partial \Delta u_{2}}{\partial x_{2}} \\
\frac{\partial \Delta u_{2}}{\partial x_{1}}+\frac{\partial \Delta u_{1}}{\partial x_{2}}
\end{array}\right]
$$

which can be related to nodal displacement increments $\Delta \mathbf{u}^{e}$ and test functions in the element $e$ through:

$$
[\Delta \varepsilon]=\overline{\mathbf{B}}_{u} \Delta \mathbf{u}^{e}, \quad[\delta \varepsilon]=\overline{\mathbf{B}}_{u} \delta \mathbf{u}^{e}
$$

where

$$
\overline{\mathbf{B}}_{u}=\left[\overline{\mathbf{D}}_{u}^{(1)} ; \overline{\mathbf{D}}_{u}^{(2)} ; \ldots ; \overline{\mathbf{D}}_{u}^{(n)}\right]
$$

with

$$
\overline{\mathbf{D}}_{u}^{(I)}=\left[\begin{array}{ll}
\frac{\partial N_{I}(\mathbf{X})}{\partial X_{1}} & 0 \\
0 & \frac{\partial N_{I}(\mathbf{X})}{\partial X_{2}} \\
\frac{\partial N_{I}(\mathbf{X})}{\partial X_{2}} & \frac{\partial N_{I}(\mathbf{x})}{\partial X_{1}}
\end{array}\right]
$$

Now we introduce the vector associated with the 2D nonsymmetric compo- 
nents of $\nabla \varepsilon$ :

$$
[\nabla \boldsymbol{\varepsilon}(\mathbf{u})]=\left[\begin{array}{l}
\nabla \varepsilon_{111} \\
\nabla \varepsilon_{112} \\
2 \nabla \varepsilon_{121} \\
2 \nabla \varepsilon_{122} \\
\nabla \varepsilon_{221} \\
\nabla \varepsilon_{222}
\end{array}\right]=\left[\begin{array}{l}
\frac{\partial^{2} u_{1}}{\partial x_{1}^{2}} \\
\frac{\partial^{2} u_{1}}{\partial x_{1} \partial x_{2}} \\
\frac{\partial^{2} u_{1}}{\partial x_{1} \partial x_{2}}+\frac{\partial^{2} u_{2}}{\partial x_{1}^{2}} \\
\frac{\partial^{2} u_{1}}{\partial x_{2}^{2}}+\frac{\partial^{2} u_{2}}{\partial x_{1} \partial x_{2}} \\
\frac{\partial^{2} u_{2}}{\partial x_{1} \partial x_{2}} \\
\frac{\partial^{2} u_{2}}{\partial x_{2}^{2}}
\end{array}\right] .
$$

Then the associated vectors $[\nabla \boldsymbol{\varepsilon}(\Delta \mathbf{u})]$ and $[\nabla \boldsymbol{\varepsilon}(\delta \mathbf{u})]$ are related to displacement increments and test functions through:

$$
[\nabla \boldsymbol{\varepsilon}(\Delta \mathbf{u})]=\tilde{\mathbf{B}}_{u} \Delta \mathbf{u}^{e}, \quad[\nabla \boldsymbol{\varepsilon}(\delta \mathbf{u})]=\tilde{\mathbf{B}}_{u} \delta \mathbf{u}^{e},
$$

where

$$
\tilde{\mathbf{B}}_{u}=\left[\tilde{\mathbf{D}}_{u}^{(1)} ; \tilde{\mathbf{D}}_{u}^{(2)} ; \ldots ; \tilde{\mathbf{D}}_{u}^{(n)}\right]
$$

with

$$
\tilde{\mathbf{D}}_{u}^{(I)}=\left[\begin{array}{ll}
\frac{\partial^{2} N_{I}(\mathbf{x})}{\partial x_{1}^{2}} & 0 \\
\frac{\partial^{2} N_{I}(\mathbf{x})}{\partial x_{1} \partial x_{2}} & 0 \\
\frac{\partial^{2} N_{I}(\mathbf{x})}{\partial x_{I} \partial x_{2}} & \frac{\partial^{2} N_{I}(\mathbf{x})}{\partial x_{1}^{2}} \\
\frac{\partial^{2} N_{I}(\mathbf{x})}{\partial x_{2}^{2}} & \frac{\partial^{2} N_{I}(\mathbf{x})}{\partial x_{1} \partial x_{2}} \\
0 & \frac{\partial^{2} N_{I}(\mathbf{x})}{\partial x_{1} \partial x_{2}} \\
0 & \frac{\partial^{2} N_{I}(\mathbf{x})}{\partial x_{2}^{2}}
\end{array}\right]
$$

We then introduce the vector containing the components of $\mathcal{S}^{\prime}$ :

$$
\left[\mathcal{S}^{\prime}\right]=\left[\begin{array}{c}
\mathcal{S}_{111}^{\prime} \\
\mathcal{S}_{112}^{\prime} \\
\mathcal{S}_{121}^{\prime} \\
\mathcal{S}_{122}^{\prime} \\
\mathcal{S}_{221}^{\prime} \\
\mathcal{S}_{222}^{\prime}
\end{array}\right]
$$


From $(190),\left[\mathcal{S}^{\prime}\right]$ is related to $[\nabla \varepsilon(\mathbf{u})]$ through:

$$
\left[\mathcal{S}^{\prime}\right]=\mathbf{T}^{\prime}[\nabla \varepsilon(\mathbf{u})]+\mathbf{U} \mathbf{p}
$$

where

$$
\mathbf{T}^{\prime}=g\left[\begin{array}{cccccc}
1 & 0 & 0 & 1 / 2 & 0 & 0 \\
0 & 0 & 0 & 0 & 0 & 0 \\
0 & 0 & 1 / 4 & 0 & 0 & 1 / 2 \\
1 / 2 & 0 & 0 & 1 / 4 & 0 & 0 \\
0 & 0 & 0 & 0 & 0 & 0 \\
0 & 0 & 1 / 2 & 0 & 0 & 1
\end{array}\right], \quad \mathbf{U}=f\left[\begin{array}{cc}
1 & 0 \\
0 & 0 \\
0 & 1 / 2 \\
1 / 2 & 0 \\
0 & 0 \\
0 & 1
\end{array}\right] .
$$

Introducing the different above discretization expressions in (198) and (11.3), we finally obtain the linear system of equations in the form:

$$
\left[\begin{array}{cc}
\tilde{\mathbf{K}}_{11} & \tilde{\mathbf{K}}_{12} \\
\tilde{\mathbf{K}}_{21} & \tilde{\mathbf{K}}_{22}
\end{array}\right]\left[\begin{array}{c}
\Delta \boldsymbol{\phi} \\
\Delta \mathbf{u}
\end{array}\right]=-\left[\begin{array}{l}
\mathbf{r}_{1}^{\prime} \\
\mathbf{r}_{2}^{\prime}
\end{array}\right]
$$

where

$$
\begin{aligned}
\tilde{\mathbf{K}}_{11} & =\int_{V} \epsilon \mathbf{B}_{\phi}^{T} \mathbf{B}_{\phi} d \Omega \\
\tilde{\mathbf{K}}_{12} & =\int_{V} \mathbf{B}_{\phi}^{T} f\left(\epsilon-\epsilon_{0}\right) \hat{\mathbf{I}} \hat{\mathbf{B}}_{u} d \Omega .
\end{aligned}
$$

Before providing the expressions for $\tilde{\mathbf{K}}_{21}$ and $\tilde{\mathbf{K}}_{22}$, we express the residuals $\mathbf{r}_{1}^{\prime}$ and $\mathbf{r}_{2}^{\prime}$ in what follows.

The residual $R_{1}^{\prime}$ is such that:

$$
R_{1}^{\prime}=\left[\delta \phi^{e}\right]^{T} \mathbf{r}_{1}^{\prime}
$$

with

$$
\begin{aligned}
& \mathbf{r}_{1}^{\prime}=\int_{V} \epsilon \mathbf{B}_{\phi}^{T} \mathbf{B}_{\phi}\left[\phi^{e}\right]^{k} d \Omega+\int_{V} f\left(\epsilon-\epsilon_{0}\right) \mathbf{B}_{\phi}^{T} \overline{\mathbf{I}} \tilde{\mathbf{B}}_{u}\left[\mathbf{u}^{e}\right]^{k} d \Omega \\
& -\int_{V} \mathbf{B}_{\phi}^{T} \mathbf{p}^{0} d \Omega-\int_{\partial V} \mathbf{N}_{\phi}^{T} D_{n}^{b} d \Gamma-\int_{V} \mathbf{N}_{\phi}^{T} \rho^{e} d \Omega .
\end{aligned}
$$


with

$$
\overline{\mathbf{I}}=\left[\begin{array}{llllll}
1 & 0 & 0 & 1 / 2 & 0 & 0 \\
0 & 0 & 1 / 2 & 0 & 0 & 1
\end{array}\right]
$$

The residual $R_{2}^{\prime}$ is such that:

$$
R_{2}^{\prime}=\left[\delta \mathbf{u}^{e}\right]^{T} \mathbf{r}_{2}^{\prime}
$$

with

$$
\begin{aligned}
& \mathbf{r}_{2}^{\prime}=\int_{\Omega} \overline{\mathbf{B}}_{u}^{T} \mathbf{C}^{e l} \overline{\mathbf{B}}_{u}\left[\mathbf{u}^{e}\right]^{k} d \Omega+\int_{\Omega} \tilde{\mathbf{B}}_{u}^{T}\left[\mathcal{S}^{\prime}\right] d \Omega+\int_{\Omega} \overline{\mathbf{B}}_{u}^{T}\left[\tilde{\boldsymbol{\sigma}}_{M W}\right] d \Omega \\
& -\int_{\Omega} \mathbf{N}_{u}^{T} \mathbf{f} d \Omega-\int_{\partial \Omega_{F}} \mathbf{N}_{u}^{T} \mathbf{t}^{e} d \Gamma
\end{aligned}
$$

with

$$
\begin{aligned}
& {\left[\tilde{\boldsymbol{\sigma}}_{M W}\right]=-\int_{\Omega} \frac{1}{2}\left\{\epsilon_{0}\left|\mathbf{e}^{k}\right|^{2}+\frac{1}{\left(\epsilon-\epsilon_{0}\right)}\left|\tilde{\mathbf{p}}^{k}\right|^{2}\right\} \mathbf{1}+\tilde{\mathbf{Z}},} \\
& \left|\mathbf{e}^{k}\right|^{2}=\left[\boldsymbol{\phi}^{e}\right]^{k^{T}} \mathbf{B}_{\phi}^{T} \mathbf{B}_{\phi}\left[\boldsymbol{\phi}^{e}\right]^{k}
\end{aligned}
$$

and

$$
\left|\tilde{\mathbf{p}}^{k}\right|^{2}=\left[\tilde{\mathbf{p}}^{k}\right]^{T} \tilde{\mathbf{p}}^{k}
$$

with

$$
\tilde{\mathbf{p}}^{k}=-\left(\epsilon-\epsilon_{0}\right)\left[f \hat{\mathbf{I}} \tilde{\mathbf{B}}_{u}\left[\mathbf{u}^{e}\right]^{k}+\mathbf{B}_{\phi}\left[\boldsymbol{\phi}^{e}\right]^{k}\right] .
$$

Above, the vector $\tilde{\mathbf{Z}}$ is given by:

$$
\tilde{\mathbf{Z}}=\left[\begin{array}{l}
Z_{11} \\
Z_{22} \\
\frac{1}{2}\left(Z_{12}+Z_{21}\right)
\end{array}\right],
$$

where

$$
\begin{aligned}
& \mathbf{Z}=\left\{\mathbf{e}^{k}\right\}\left\{\mathbf{d}^{k}\right\}^{T}, \quad \mathbf{e}^{k}=-\mathbf{B}_{\phi}\left[\boldsymbol{\phi}^{e}\right]^{k} \\
& \mathbf{d}^{k}=-\epsilon \mathbf{B}_{\phi}\left[\boldsymbol{\phi}^{e}\right]^{k}-f\left(\epsilon-\epsilon_{0}\right) \hat{\mathbf{I}} \tilde{\mathbf{B}}_{u}\left[\mathbf{u}^{e}\right]^{k}+\mathbf{p}^{0}
\end{aligned}
$$


and

$$
\mathbf{1}=\left[\begin{array}{l}
1 \\
1 \\
0
\end{array}\right]
$$

The tangent matrices $\tilde{\mathbf{K}}_{21}$ and $\tilde{\mathbf{K}}_{22}$ can then be written as

$$
\tilde{\mathbf{K}}_{21}=\int_{\Omega}-\overline{\mathbf{B}}_{u}^{T} \mathbf{1}\left(\epsilon_{0} \mathbf{a}+\left(\epsilon-\epsilon_{0}\right) \mathbf{b}\right)+\Xi^{(1)} d \Omega
$$

with

$$
\begin{aligned}
& \mathbf{a}=\left[\boldsymbol{\phi}^{k}\right]^{T} \mathbf{B}_{\phi}^{T} \mathbf{B}_{\phi} \\
& \mathbf{b}=\left[\boldsymbol{\phi}^{k}\right]^{T} \mathbf{B}_{\phi}^{T}\left(f \hat{\mathbf{I}} \tilde{\mathbf{B}}_{u}\left[\mathbf{u}^{e}\right]^{k}+\mathbf{B}_{\phi}\left[\boldsymbol{\phi}^{e}\right]^{k}\right)
\end{aligned}
$$

and

$$
\Xi^{(1)}=\left[\begin{array}{l}
\mathbf{b}_{\phi}^{1}\left(\epsilon\left[\boldsymbol{\phi}^{e}\right]^{k} \mathbf{b}_{\phi}^{1}-d_{1}\right) \\
\mathbf{b}_{\phi}^{2}\left(\epsilon\left[\boldsymbol{\phi}^{e}\right]^{k} \mathbf{b}_{\phi}^{2}-d_{2}\right) \\
\frac{1}{2}\left[\mathbf{b}_{\phi}^{1}\left(\epsilon\left[\boldsymbol{\phi}^{e}\right]^{k} \mathbf{b}_{\phi}^{2}-d_{2}\right)+\mathbf{b}_{\phi}^{2}\left(\epsilon\left[\boldsymbol{\phi}^{e}\right]^{k} \mathbf{b}_{\phi}^{1}-d_{1}\right)\right]
\end{array}\right]
$$

where $\mathbf{b}_{\phi}^{i}$ is the $i-t h$ row of the matrix $\mathbf{B}_{\phi}$ and $d_{i}$ is the $i-t h$ component of d, and

$$
\begin{aligned}
& \tilde{\mathbf{K}}_{22}=\int_{\Omega} \overline{\mathbf{B}}_{u}^{T} \mathbf{C}^{e l} \mathbf{B}_{u} d \Omega+\int_{\Omega} \tilde{\mathbf{B}}_{u}^{T} \mathbf{T}^{\prime} \tilde{\mathbf{B}}_{u}-f\left(\epsilon-\epsilon_{0}\right) \tilde{\mathbf{B}}_{u}^{T} \mathbf{U} \hat{\mathbf{I}} \tilde{\mathbf{B}}_{u} d \Omega \\
& -\int_{\Omega} \overline{\mathbf{B}}_{u}^{T}\left(\mathbf{1} \mathbf{c}^{T}-\Xi^{(2)}\right) d \Omega
\end{aligned}
$$

with

$$
\mathbf{c}=f\left(\epsilon-\epsilon_{0}\right) \tilde{\mathbf{B}}_{u}^{T} \overline{\mathbf{I}}^{T}\left(f \hat{\mathbf{I}} \tilde{\mathbf{B}}_{u}\left[\mathbf{u}^{e}\right]^{k}+\mathbf{B}_{\phi}\left[\boldsymbol{\phi}^{e}\right]^{k}\right)
$$

and

$$
\Xi^{(2)}=\left[\begin{array}{l}
\mathbf{b}_{\phi}^{1}\left[\boldsymbol{\phi}^{e}\right]{ }^{k \hat{\mathbf{i}}^{1}} \tilde{\mathbf{B}}_{u} \\
\mathbf{b}_{\phi}^{2}\left[\boldsymbol{\phi}^{e}\right]{ }^{k} \hat{\mathbf{i}}^{2} \tilde{\mathbf{B}}_{u} \\
\frac{1}{2}\left(\mathbf{b}_{\phi}^{1}\left[\boldsymbol{\phi}^{e}\right]^{k} \hat{\mathbf{i}}^{2}+\mathbf{b}_{\phi}^{2}\left[\boldsymbol{\phi}^{e}\right] \hat{\mathbf{i}}^{1}\right) \tilde{\mathbf{B}}_{u}
\end{array}\right]
$$

where $\hat{\mathbf{i}}^{j}$ is the $j-t h$ line of $\overline{\mathbf{I}}$. 


\section{References}

[1] A. Abdollahi, P.C Christian, M.D. Daniel, M. Arroyo, and A.I. Irene. Computational evaluation of the flexoelectric effect in dielectric solids. J. Appl. Phys., 116(9):093502, 2014.

[2] J. Aboudi. Micro-electromechanics of soft dielectric matrix composites. Int. J. Solids Struct., 64-65:30-41, 2015.

[3] R. Ahluwalia, A. K. Tagantsev, P. Yudin, N. Setter, N. Ng, and D. J. Srolovitz. Influence of flexoelectric coupling on domain patterns in ferroelectrics. Phys. Rev. B, 89:174105, 2014.

[4] E. Amanatidou and N. Aravas. Mixed finite element formulations of strain gradient elasticity problems. Comput. Meth. Appl. Mech. Eng., 191:1723-1751, 2002 .

[5] J.Y. Fu anf W. Zhu, N. Li, and L.E. Cross. Experimental studies of the converse flexoelectric effect induced by inhomogeneous electric field in a barium strontium titanate composition. J. Appl. Phys., 100(2):024112, 2006.

[6] F. Carpi, S. Bauer, and D. De Rossi. Stretching dielectric elastomers performance. Science, 330:1759-1761, 2010.

[7] Y. Chen, M. Pani, F. Taddei, and C. Mazzà. Large-scale finite element analysis of human cancellous bone tissue micro computer tomography data: a convergence study. J Biomech. Eng.-T. ASME, 136(10):101013, 2014.

[8] B. Chu and D.R. Salem. Flexoelectricity in several thermoplastic and thermosetting polymers. Appl. Phys. Lett., 101:103905, 2012.

[9] L.E. Cross. Flexoelectric effects: Charge separation in insulating solids subjected to elastic strain gradients. J. Mater. Sci., 41(1):53-63, 2006.

[10] Q.D. Deng, L.P. Liu, and P. Sharma. Flexoelectricity in soft materials and biological membranes. J. Mech. Phys. Solids, 62:209-227, 2014.

[11] V. Dominguez and F.J. Saya. A simple Matlab implementation of the Argyris element. ACM T. Math. Software, 35(2):16, 2008.

[12] A. Dorfmann and R.W. Ogden. Nonlinear electroelasticity. Acta Mech., 174:167-183, 2005.

[13] G. Gallone, F. Galantini, and F. Carpi. Perspective for new dielectric elastomers with improved electromechanical actuation performance: composites versus blends. Polym. Int., 59:400-406, 2010.

[14] G.A. Holzapfel. Nonlinear solid mechanics. Wiley, 2000.

[15] S. Y. Hu and L. Q. Chen. A phase-field model for evolving microstructures with strong elastic inhomogeneity. Acta Mater., 49:1879, 2001. 
[16] J. Huang, T. Lu anf J. Zhu, D.R. Clarke, and Z. Suo. Tuni-directional actuation in dielectric elastomers achieved by fiber stiffening. Appl. Phys. Lett., 100:211901, 2012.

[17] T.A. Gisby I.A. Anderson, B.M. O'Brien T.G. McKay, and E.P. Calius. Multifunctional dielectric elastomer artificial muscles for soft and smart machines. $J$. Appl. Phys., 112:041101, 2012.

[18] Y. Bazilevs J. Cotterel, T.J.R. Hughes. Isogeometric analysis: Toward integration of CAD and FEA. Wiley, 2009.

[19] J.P. Jaric, D. Kuzmanovic, and Z.Golubovic. On tensors of elasticity. Theor. Appl. Mech., 35:119-136, 2008.

[20] S.M. Kogan. Piezoelectric effect during inhomogeneous deformation and acoustic scattering of carriers in crystals. Sov. Phys. Solid Stat, 5(10), 1964.

[21] S. Krichen and P. Sharma. Flexoelectricity: A perspective on an unusual electromechanical coupling. J. Appl. Mech., 83:030801-1, 2016.

[22] W. Li and C.M. Landis. Deformation and instabilities in dielectric elastomer composites. Smart Mater. Struct., 21:094006, 2012.

[23] L.P. Liu. An energy formulation of continuum magneto-electro-elasticity with applications. J. Mech. Phys. Solids, 63:451-480, 2014.

[24] O. Lopez-Pamies. Elastic dielectric composites: Theory and application to particle-filled ideal dielectrics. J. Mech. Phys. Solids, 64:61-82, 2014.

[25] W. Ma and L.E. Cross. Flexoelectric polarization of barium strontium titanate in the paraelectric state. Appl. Phys. Lett., 81(18):3440-3442, 2002.

[26] W. Ma and L.E. Cross. Strain-gradient induced electric polarization in lead zirconate titanate ceramics. Appl. Phys. Lett., 82(19):3923-3925, 2003.

[27] T.G. McKay, B.M. O'Brien, E.P. Calius, and I.A. Anderson. Soft generators using dielectric elastomers. Appl. Phys. Lett., 98:142903, 2011.

[28] R.M. McMeeking and C.M. Landis. Electroelastic forces and stored energy for deformable dielectric materials. J. Appl. Mech, 72:581-590, 2005.

[29] H.S. Park, Z. Suo anf J. Zhuo, and P.A. Klein. A dynamic finite element method for inhomogeneous deformation and electromechanical instability of dielectric elastomer transducers. Int. J. Solids Struct., 49:2187-2194, 2012.

[30] H.S. Park and T.D. Nguyen. Viscoelastic effects on electromechanical instabilities in dielectric elastomers. Soft matter, 9(4):1031-1042, 2013.

[31] R. Pelrine, R. Kornbluh, Q.B. Pei, and J. Joseph. High-speed electrically actuated elastomers with strain greater than 100. Science, 287:836-839, 2000.

[32] A.W. Richards and G.M. Constitutive modeling of electrostrictive polymers using hyperelasticity-based approach. J. Appl. Mech., 77:014502, 2010. 
[33] N.D. Sharma, R. Maraganti, and P. Sharma. On the possibility of piezoelectric nanocomposites without using piezoelectric materials. J. Mech. Phys. Solids, $55: 2328-2350,2007$.

[34] J.Y. Shu, W.E. King, and N.A. Fleck. Finite elements form materials with strain gradient effects. Int. J. Num. Meth. Eng., 44:373-391, 1999.

[35] Z. Suo, X. Zhao, and W.H. Greene. A nonlinear theory of deformable dielectrics. J. Mech. Phys. Solids, 56:467-486, 2008.

[36] K.Y. Volokh. On electromechanical coupling in elastomers. J. Appl. Mech, 79:044507, 2012.

[37] Q. W. Wang, Z.G. Suo, and X.H. Zhao. Bursting drops in solid dielecrtics caused by high voltages. Nature comm., 3:1157, 2012.

[38] Y.U. Wang, Y.M.M. Jin, and A.G. Khachaturyan. Phase field microelasticity theory and modeling of elastically and structurally inhomogeneous solid. $J$. Appl. Phys., 92:1351, 2002.

[39] L. Yang and K. Dayal. A completely iterative method for the infinite domain electrostatic problem with nonlinear dielectric media. J. Comput. Phys., 230:7821-7829, 2011.

[40] P.V. Yudin and A.K. Tagantsev. Fundamentals of flexoelextricity in solids. Nanotechnology, 24:432001, 2013.

[41] X. Zhao, W. Hong, and Z. Suo. Electromechanical hysteresis and coexistent states in dielectric elastomers. Phys. Rev. B, 76:134113, 2007.

[42] X. Zhao and Z. Suo. Electrostriction in elastic dielectrics undergoing large deformation. J. Appl. Phys., 104:123530, 2008.

[43] X. Zhao and Z. Suo. Theory of dielectric elastomers capable of giant deformation of actuation. Phys. Rev. Lett, 104:178302, 2010.

[44] G.P. Zubko, A.R. Catalan, P. Buckley, L. Welche, and J.F. Scott. Straingradient induced polarization in SrTiO3 single crystals. Phys. Rev. Lett., 99(16):167601, 2007. 